\title{
Mechanisms of up-regulation of Ubiquitin-Proteasome activity in the absence of NatA dependent $\mathrm{N}$-terminal acetylation
}

Ilia Kats ${ }^{1,2}$, Marc Kschonsak ${ }^{1,3}$, Anton Khmelinskii ${ }^{4}$, Laura Armbruster ${ }^{1,5}$, Thomas Ruppert $^{1}$ and Michael Knop ${ }^{1,6,{ }^{*}}$

${ }^{1}$ Zentrum für Molekulare Biologie der Universität Heidelberg (ZMBH), DKFZ-ZMBH Alliance, Im Neuenheimer Feld 282, 69120 Heidelberg, Germany.

${ }^{2}$ present address: German Cancer Research Center (DKFZ), Im Neuenheimer Feld 280, 69120 Heidelberg, Germany

${ }^{3}$ present address: Department of Structural Biology, Genentech Inc., South San Francisco, CA, USA.

${ }^{4}$ Institute of Molecular Biology (IMB), Ackermannweg 4, 55128 Mainz, Germany.

${ }^{5}$ present address: Centre for Organismal Studies (COS), Im Neuenheimer Feld 360, 69120 Heidelberg, Germany

${ }^{6}$ Deutsches Krebsforschungszentrum (DKFZ), DKFZ-ZMBH Alliance, Im Neuenheimer Feld 280, 69120 Heidelberg, Germany.

* corresponding author: m.knop@zmbh.uni-heidelberg.de

\begin{abstract}
$\mathrm{N}$-terminal acetylation is a prominent protein modification and inactivation of $\mathrm{N}$-terminal acetyltransferases (NATs) cause protein homeostasis stress. Using multiplexed protein stability (MPS) profiling with linear ubiquitin fusions as reporters for the activity of the ubiquitin proteasome system (UPS) we observed increased UPS activity in NatA, but not NatB or NatC mutants. We find several mechanisms contributing to this behavior. First, NatA-mediated acetylation of the N-terminal ubiquitin independent degron regulates the abundance of Rpn4, the master regulator of the expression of proteasomal genes. Second, the abundance of several E3 ligases involved in degradation of UFD substrates is increased in cells lacking NatA. Finally, we identify the $\mathrm{E} 3$ ligase Tom1 as a novel chain elongating enzyme (E4) involved in the degradation of linear ubiquitin fusions via the formation of branched K11 and K29 ubiquitin chains, independently of the known E4 ligases involved in UFD, leading to enhanced ubiquitination of the UFD substrates.
\end{abstract}

\section{Introduction}

Selective protein degradation is essential for proteome homeostasis, to remove unnecessary or abnormal proteins as part of quality control pathways or in response to changes in the environment. In eukaryotes the bulk of selective protein degradation is handled by the ubiquitin-proteasome system (UPS). Substrates of the UPS are recognized through features known as degradation signals or degrons (Ravid and Hochstrasser, 2008), ubiquitinated by E3 ubiquitin ligases typically on lysine side chains, and finally degraded by the proteasome (Finley et al., 2012; Hershko and Ciechanover, 1998).

Global activity of the UPS is tightly regulated and responds to environmental challenges such as heat stress, DNA damage or cytotoxic compounds, which can damage or induce misfolding of proteins (Hahn et al., 2006). In the budding yeast Saccharomyces cerevisiae, the transcription factor Rpn4 is a master regulator of proteasome capacity. It trans-activates promoters of all proteasomal subunits and several other proteins of the UPS (Mannhaupt et al., 1999; Shirozu et al., 2015). Expression of Rpn4 is in turn regulated by several stressinduced transcription factors such as Hsf1 and Yap1 (Hahn et al., 2006). 
In addition to global regulation of the UPS that affects the entire proteome, selective degradation of specific proteins can be induced through post-translational modifications creating or exposing degradation signals. $\mathrm{N}$-degrons that target for degradation via an $\mathrm{N}$ terminal destabilizing residue can be formed by specific endoproteolytic cleavage. For example, cohesin cleavage by separase at the metaphase-anaphase transition induces degradation of the $\mathrm{C}$-terminal fragment by the $\mathrm{Arg} / \mathrm{N}$-end rule pathway that recognizes the newly exposed $\mathrm{N}$-terminal residue as a degradation signal (Rao et al., 2001).

Na-terminal acetylation of proteins (Nt-acetylation) is a co-translational modification catalyzed by ribosome-associated Na-terminal acetyltransferase (NAT) complexes. Three NATs, NatA, $\mathrm{NatB}$, and NatC, are responsible for the acetylation of $50-90 \%$ of all protein $\mathrm{N}$-termini in yeast and human cells (Aksnes et al., 2016; Starheim et al., 2012). These NATs differ in their substrate specificity. NatA acetylates the small residues $(S, A, V, C, G)$ after they have been exposed at the $\mathrm{N}$-terminus through cleavage of the initiator methionine (iMet) by methionine aminopeptidases (MetAPs). NatB and NatC acetylate the iMet if it is followed by a polar residue (one of $(D, E, N, Q)$ ) or a large hydrophobic residue (one of $(F, L, I, W)$ ), respectively. The identity of the first two $\mathrm{N}$-terminal residues is however not sufficient to trigger $\mathrm{Nt}$-acetylation, and numerous proteins lack this modification despite being potential NAT substrates according to their primary sequence (Aksnes et al., 2016).

$\mathrm{Nt}$-acetylation of nascent chains is a prevalent protein modification affecting the majority of all proteins and it has been implicated in a multitude of cellular processes. Deletion of the major $\mathrm{N}$-acetyl transferase genes leads to pleiotropic effects with distinct influences on the physiology and cellular proteostasis of $S$. cerevisiae. For NatA several individual targets are known where $\mathrm{Nt}$-acetylation functions in mediating protein-protein interactions, prevention of incorrect protein secretion, protein folding and degradation. This includes key transcriptional regulators as well as protein folding machinery or structural components of the cytoskeleton (Aksnes et al., 2016; Friedrich et al.), therefore it is not surprising that the attribution of specific functions to Na-acetylated $\mathrm{N}$-termini is not possible. Another important point is the question to what extent Nt-acetylation is subject to specific regulation, e.g. via regulation of the activity of the individual NATs. While there is some evidence from plants that NatA activity can be regulated as a function of drought stress (Linster et al., 2015), in yeast no clear reports about specific regulation of NATs exist. This is consistent with the observation that Nt-acetylation appears to be irreversible and that is hardly affected by reduced Acetyl-CoA levels (Varland et al., 2018).

$\mathrm{Nt}$-acetylation was proposed to act as a degradation signal (Hwang et al., 2010b; Shemorry et al., 2013) and Nt-acetylated $\mathrm{N}$-termini are thought to be recognized and ubiquitinated by specific E3 ligases of the Ac/N-end rule pathway. The universality of this pathway is debatable, because acetylation is not a self-sufficient degron and the involved E3 ligases recognize a broad palette of N-degrons independent on Nt-acetylation (Friedrich et al.; Gawron et al., 2016; Kats et al., 2018; Zattas et al., 2013). Still, Nt-acetylation can be part of N-degrons that contain adjacent sequence motifs (Hwang et al., 2010b; Shemorry et al., 2013). In recognition of the fact that Nt-acetylation is not a general degron, it was finally proposed to refer to ' $\mathrm{N}$-terminal degrons', and to avoid the wording 'N-end rule' (Varshavsky, 2019), in favor of specific terminology that refers to the individualistic nature of each $\mathrm{N}$-terminal degron. This is even more important given that accumulating evidence suggests that $\mathrm{N}$-acetylation can fulfill the exact opposite function: as a protein stabilizing modification. First, Nt-acetylation can prevent direct ubiquitylation of the Na amino group of proteins (Caron et al., 2005; Hershko et al., 1984; Kuo et al., 2004). This may be the underlying mechanism how Nt-acetylation protects the Derlin protein Der1 from degradation by the associated E3 ligase Hrd1 (Zattas et al., 2013). 
Acetylation can also protect $\mathrm{N}$-termini from non-canonical processing by aminopeptidases, i.e. methionine aminopeptidases 1 and 2 (MAP1/2), which, in the absence of Nt-acetylation, can remove the initiator methionine (iMet) form the nascent chain. This leads to the exposure of the second residue, which in the case of NatB and NatC N-termini will lead to the exposure of an Arg/N-degron that can targets the protein for Ubr1 dependent degradation (Kats et al., 2018; Nguyen et al., 2018).

The yeast genome encodes several linear ubiquitin fusion proteins which serve as a source of free ubiquitin, since the $\mathrm{N}$-terminal ubiquitin moiety is usually co-translationally cleaved off by endogenous deubiquitinating enzymes (DUBs) (Amerik and Hochstrasser, 2004). Linear ubiquitin fusions that escape DUB cleavage or that are generated post-translationally by ubiquitination of the $\mathrm{Na}$ group of the first amino acid residue of a protein can be further ubiquitinated by E3 ligases of the ubiquitin-fusion degradation (UFD) pathway using conventional lysine- $\varepsilon$-amino-specific linkage on at least one of the seven lysine residues of the $\mathrm{N}$-terminal ubiquitin moiety and degraded by the proteasome. In yeast, Ufd4 is the major E3 ligase of the UFD pathway (Johnson et al., 1995), while the accessory E3 ligases Ufd2 and Ubr1 promote degradation by acting as chain elongating enzymes (E4 ligases) (Hwang et al., 2010a; Koegl et al., 1999). The UFD pathway is conserved in humans, where it is composed of the Ufd4 ortholog TRIP12 and the Ufd2 orthologs UFD2a and UFD2b (Park et al., 2009). The pathway was first identified in yeast using artificial substrates consisting of linear ubiquitin fusions (UbiG76V) that are resistant to cleavage by DUBs (Johnson et al., 1995). Such UFD substrates were subsequently used as a high-throughput-compatible readout of proteasome activity (Dantuma et al., 2000; Stack et al., 2000). However, endogenous substrates of the UFD pathway have proven difficult to identify, and only few are known to date. Nevertheless, mammalian cells possess the E2 conjugating enzyme Ube2w that monoubiquitinates $\mathrm{N}$ terminal residues if they are followed by an intrinsically disordered sequence (Scaglione et al., 2013; Tatham et al., 2013; Vittal et al., 2014) as well as the E3 ligase LUBAC that assembles linear M1-linked ubiquitin chains and was implicated in immune signaling (Fiil et al., 2013; Gerlach et al., 2011; Tokunaga et al., 2009). However, to the best of our knowledge, the origin of the N-terminal ubiquitin moiety in known endogenous UFD substrates has not been investigated, and all known instances of N-terminal ubiquitination by LUBAC or Ube2w do not induce degradation of the substrate, but rather mediate protein-protein interactions or activate signaling cascades (Rittinger and Ikeda, 2017). N-terminal ubiquitination has been suggested to be regulated by Nt-acetylation, as both modifications involve the same amino group (Caron et al., 2005; McDowell and Philpott, 2013).

We have developed multiplexed protein stability (MPS) profiling, a quantitative and highthroughput compatible method that enables the degradation profiling of large peptide libraries using fluorescence activated cell sorting (FACS) and analysis of enriched fractions by deep sequencing (Kats et al., 2018). We used MPS profiling to explore the degron propensity of native and non-native $\mathrm{N}$-termini and a large fraction of the yeast $\mathrm{N}$-termini ( $\mathrm{N}$-terminome) (Kats et al., 2018). In this work we explore the influence of NatA on protein degradation in the budding yeast $S$. cerevisiae starting with the observation that artificial UFD substrates are degraded faster in NatA-deficient cells. Using screening and targeted we describe a role for $\mathrm{Nt}$-acetylation on regulation of UPS activity via Rpn4 and we investigate how the abundance of several E3 and E4 ubiquitin ligases is influenced by NatA and how this contributes to UFD. We furthermore identify Tom1 as a novel ubiquitin chain-elongating enzyme (E4) of the UFD pathway and using in vivo and in vitro assays we investigate ubiquitination by Tom1. Altogether, our data provide new insights into the molecular processes governing UPS activity 
regulation in the absence of NatA activity, emphasizing the importance of NatA for cellular protein homeostasis.

\section{Results}

\section{NatA affects turnover of UFD substrates}

We performed a systematic survey of degrons in protein N-termini using linear ubiquitin fusion reporter constructs (Kats et al., 2018). These reporters consisted of an N-terminal ubiquitin followed by two variable residues ( $\mathrm{X}$ and $\mathrm{Z}$ ), a linker sequence $(\mathrm{eK})$ and a tandem fluorescent protein timer ( $\mathrm{tFT}$ ). The tFT reports on protein stability independently of expression through the intensity ratio of the slow maturing mCherry and the fast maturing sfGFP fluorescent proteins, which increases as a function of protein half-life in steady state (Khmelinskii et al., 2012; Khmelinskii et al., 2016). In the course of that study we observed that reporters with a proline residue immediately following the ubiquitin moiety (Ubi-PZ-tFT reporters) exhibited increased turnover in strains lacking the N-terminal acetyltransferase NatA (Fig 1A), whereas no destabilization was observed in NatB and NatC mutants (see Supplementary Figure S3 in (Kats et al., 2018)). The N-terminal ubiquitin moiety is usually co-translationally cleaved by endogenous deubiquitinating enzymes (DUBs) (Bachmair et al., 1986), which enables the exposure of non-native amino acid residues at the $\mathrm{N}$-terminus of the reporter protein. However, a proline residue located directly after ubiquitin impairs cleavage of the ubiquitin moiety by DUBs. Such linear ubiquitin fusions are rapidly degraded by the ubiquitin fusion degradation (UFD) pathway (Johnson et al., 1995), primarily through the action of the ubiquitin E3 ligases Ufd4 and Ubr1 (Hwang et al., 2010a). In contrast, cleaved Ubi-PZ-tFT reporters with an exposed $\mathrm{N}$-terminal proline are stable (Bachmair et al., 1986; Bachmair and Varshavsky, 1989).

To understand how NatA affects turnover of Ubi-PZ-tFT reporters, we first confirmed that these reporters are affected by deletion of the catalytic NatA subunit NAA10 using cycloheximide chase experiments. These immunoblots indicated that abundance and/or degradation of an uncleaved Ubi-PP-tFT reporter are influenced by deletion of NAA10, the catalytic subunit of NatA (Fig 1B). These results can be explained either by accelerated degradation of uncleaved Ubi-PZ-tFT reporters or by impaired DUB activity in the naa10 $\Delta$ mutant. In DUB-impaired cells, a larger fraction of Ubi-PZ-tFT reporters would remain uncleaved, and rapid degradation of uncleaved Ubi-PZ-tFT reporters by the UFD pathway would account for their apparent destabilization. To distinguish between these possibilities, we investigated turnover of a noncleavable UbiG76V-tFT reporter, in which the last glycine of ubiquitin is exchanged for valine to completely prevent cleavage by DUBs (Johnson et al., 1992). Degradation of this reporter was inferred from mCherry/sfGFP ratio as measured by flow cytometry. Stability of the UbiG76V-tFT reporter in wild type yeast was at the lower end of the tFT dynamic range, therefore no clear effect of NAA10 deletion could be detected by flow cytometry (Fig 1C, pos. $1 \& 2,5 \& 6)$. As expected, this reporter was strongly stabilized in ufd $4 \Delta$ and ubr $1 \Delta$ ufd $4 \Delta$ cells. Surprisingly however, it was still degraded in these mutants and moreover, it was clearly destabilized upon deletion of $N A A 10$ (Figs 1C, pos. 3\&4, 7\&8, for a CHX chase, see Fig. S1). This suggests that NatA-dependent acceleration of UFD substrate turnover is independent of DUB activity. The results also suggest that accelerated degradation does not involve the canonical E3 ubiquitin ligases implicated in the degradation of such linear ubiquitin fusions. 
A

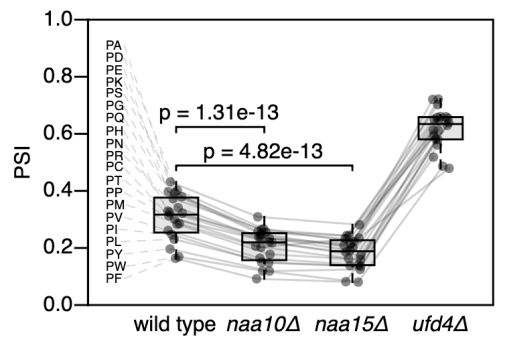

B

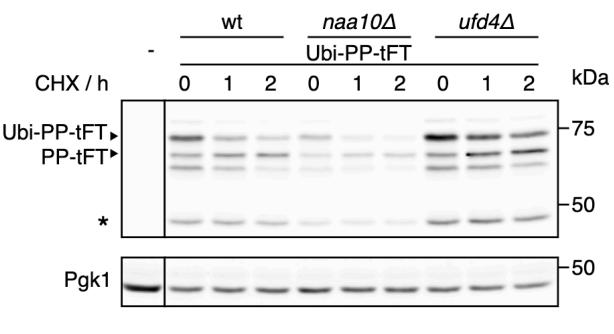

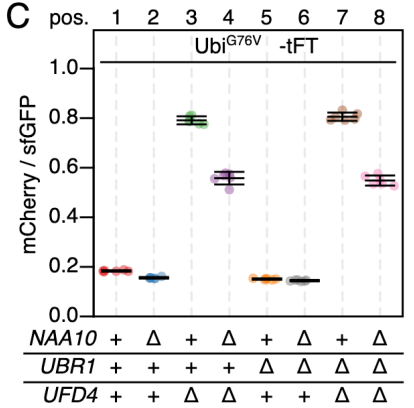

Fig 1. Accelerated degradation of linear ubiquitin fusion proteins in NatA-deficient strains.

(A) Average stability of Ubi-PZ-tFT reporters in the indicated strains. The protein stability index (PSI) is a measure of protein turnover resulting from high-throughput analysis of tFT-tagged constructs and increases as a function of the mCherry/sfGFP ratio and is therefore anticorrelated with degradation rate. Data from Kats et al. (Kats et al., 2018). Boxplots show median, $1^{\text {st }}$ and $3^{\text {rd }}$ quartile, whiskers extend to $\pm 1.5 \mathrm{x}$ interquartile range (IQR) from the box. $\mathrm{p}$ : two-sided paired t-test.

(B) Degradation of the Ubi-PP-tFT reporter after blocking translation with cycloheximide. Whole-cell extracts were separated by SDS-PAGE followed by immunoblotting with antibodies against GFP and Pgk1 as loading control. A product resulting from mCherry autohydrolysis during cell extract preparation (Gross et al., 2000) is marked $(*)$.

(C) Flow cytometry analysis of strains expressing the UbiG76V-tFT reporter. For all flow cytometry experiments, mCherry/sfGFP ratios were normalized to a stable control measured in the same strain background. Mean mCherry/sfGFP ratios and $95 \% \mathrm{Cl}$ of six replicates are plotted together with the median mCherry/sfGFP ratio of each replicate.

\section{Nt-acetylation by NatA promotes ubiquitin-independent degradation of Rpn4}

DUB-independent destabilization of the UbiG76V-tFT reporter in strains lacking the known E3s of the UFD pathway suggested that at least one additional E3 ligase involved in degradation of UFD substrates exists. While searching for this E3, we noticed that deletion of the Ubr2 E3 ligase in the ubr1 $\Delta$ ufd $4 \Delta$ background accelerated degradation of the UbiG76V-tFT reporter. This destabilization was additive to the effect of NAA10 deletion on UbiG76V-tFT reporter stability (Fig 2A, pos. 1 to 4). Ubr2 acts via the Rpn4 transcription factor to regulate expression of UPS genes. More specifically, Rpn4 possesses two degrons, a ubiquitin-dependent degron that is recognized by Ubr2, and an N-terminal ubiquitin-independent degron that is directly recognized by the 26S proteasome (Ju et al., 2004; Ju and Xie, 2004; Ju and Xie, 2006; Wang et al., 2004a) (Fig 2B). These degrons induce a negative feedback loop regulating UPS activity, such that Rpn4 abundance and consequently proteasome biogenesis are balanced to meet the proteolytic load (Xie and Varshavsky, 2001). Deletion of the Ubr2-dependent degron of Rpn4 (Rpn4 $\Delta(211-229)$ (Wang et al., 2010)) destabilized the UbiG76V-tFT reporter in the ubr1 $\Delta$ ufd $4 \Delta$ background. No further destabilization of this reporter was observed upon additional deletion of UBR2 (Fig 2A, pos. 5 to 8). This indicates that accelerated degradation of the UbiG76V-tFT reporter upon ablation of Ubr2 is due to stabilization of Rpn4. Rpn4 is a potential NatA substrate according to its primary sequence, which starts with MA. To explain the additive effect of NatA deletion on degradation of the UbiG76V-tFT reporter, we hypothesized that $\mathrm{Nt}$-acetylation of Rpn4 affects its $\mathrm{N}$-terminal ubiquitin-independent degron. Consistent with this idea, abundance of C-terminally TAP-tagged Rpn4 was strongly increased 
in the naa10 $\Delta$ mutant (Fig 2C). To test this hypothesis directly, we exploited the portability of the ubiquitin-independent degron of Rpn4 (Ha et al., 2012) and measured turnover of an Rpn4(1-80)-tFT reporter containing the N-terminal Ubi-independent degron of Rpn4 fused to the tFT. This reporter was stabilized upon deletion of $N A A 10$ (Fig 2D, Pos. 1\&2). Preventing NatA-mediated Nt-acetylation by substituting the second residue for asparagine strongly reduced stabilization of the reporter in the naa10 $\Delta$ mutant (Fig 2D, Pos. 4\&5). Instead, this Rpn4A2N(1-80)-tFT reporter, a potential target of NatB, was stabilized in naa20 $\Delta$ cells lacking the catalytic subunit of NatB (Fig 2D, Pos. 4\&6) to a similar extent as the Rpn4(1-80) reporter in naa10 $\Delta$ cells (Fig 2D, Pos. 1\&2). These results indicate that Rpn4A2N(1-80)-tFT is acetylated by NatB, and that Nt-acetylation, regardless of the NAT, promotes ubiquitinindependent degradation of Rpn4. Label-free quantitative mass spectrometry of full-length Rpn4 confirmed NatA-dependent acetylation of Rpn4 and NatB-dependent acetylation of Rpn4A2N (Fig 2E).

Next we investigated the influence of NatA on ubiquitin-independent degradation of Rpn4 in a physiological context. We performed cycloheximide chases of C-terminally TAP-tagged Rpn4 lacking its ubiquitin-dependent degron (Rpn4 $\Delta(211-229)-T A P)$. Deletion of NAA10 doubled the half-life of Rpn4 $\Delta$ (211-229-TAP), but not of Rpn4A2N, $\Delta$ (211-229-TAP) (Fig 2F and Fig S2A,B). Taken together, these results argue that NatA-mediated N-terminal acetylation of Rpn4 promotes its ubiquitin-independent degradation, thereby modulating its abundance.

To assess if Rpn4 mediates the accelerated degradation of the UbiG76V-tFT reporter in the naa10 $\Delta$ mutant, we measured turnover of this reporter in cells carrying the rpn4A2N allele using flow cytometry. Destabilization of this reporter upon deletion of NAA10 was only marginally reduced in the Rpn4A2N mutant compared to cells expressing wild type Rpn4 (Fig $2 \mathrm{G}$ ). This suggests that elevated levels of Rpn4 could contribute to, but are not solely responsible for, accelerated turnover of UFD substrates in the absence of NatA.

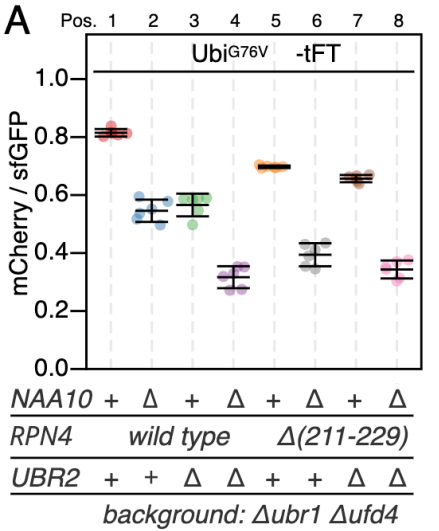

$E$

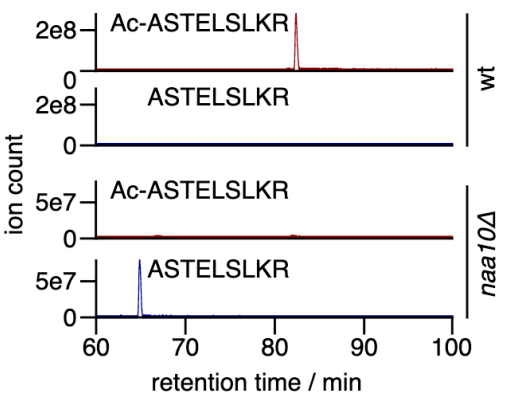

B
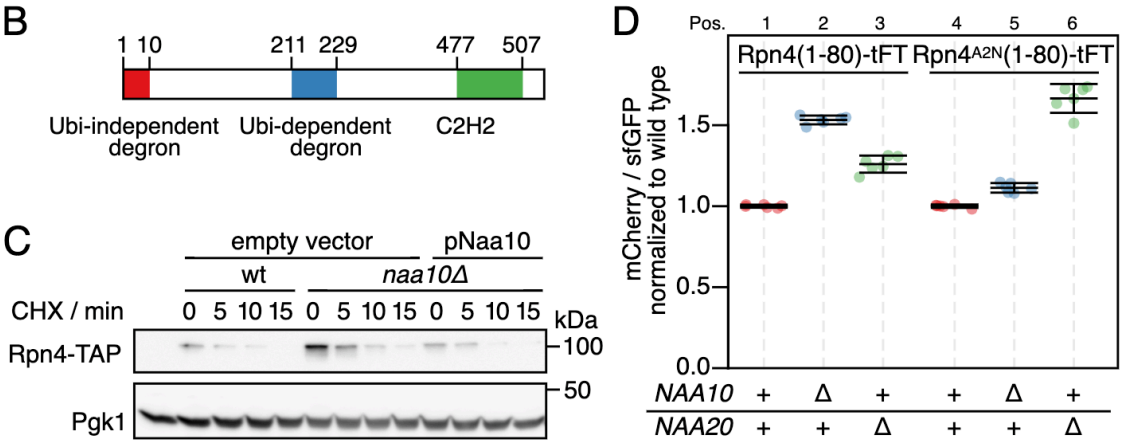

$\mathrm{F}$

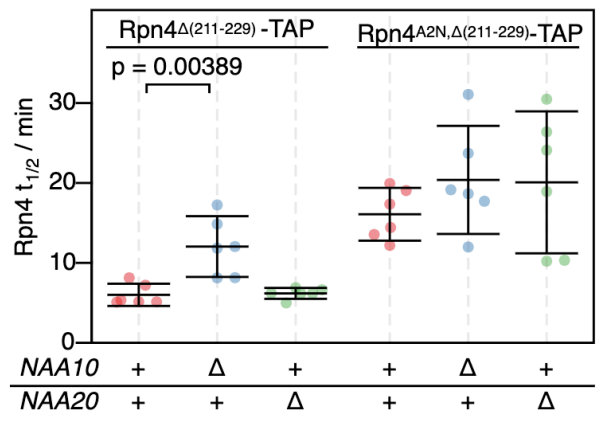

G

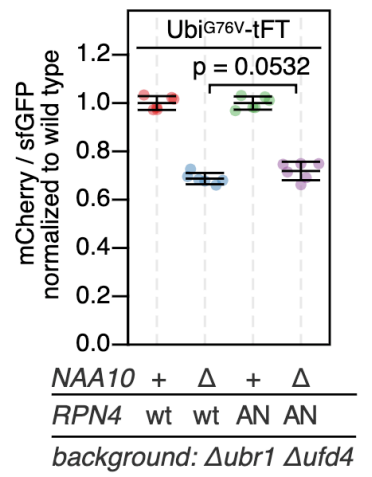




\section{Fig 2: Regulation of the ubiquitin independent degron of Rpn4 by NatA and contribution to degradation of UFD substrates.}

(A) Flow cytometry analysis of strains expressing the UbiG76V-tFT reporter.

(B) Domain architecture of Rpn4.

(C) Degradation of C-terminally TAP-tagged Rpn4 after blocking translation with cycloheximide. Whole-cell extracts were separated by SDS-PAGE followed by immunoblotting with antibodies against protein $\mathrm{A}$ and Pgk1 as loading control.

(D) Flow cytometry analysis of strains expressing the indicated Rpn4 N-terminal sequences fused to the tFT. mCherry/sfGFP ratios were normalized to the mean mCherry/sfGFP ratio of the wild type strain.

(E) Extracted ion chromatograms of Nt-acetylated and unmodified $\mathrm{N}$-terminal peptides derived from full-length Rpn4 variants obtained by label-free mass spectrometry.

(F) Half-lives of C-terminally TAP-tagged Rpn4 variants estimated by cycloheximide chase. Mean half-lives and $95 \% \mathrm{Cl}$ of six replicates are plotted together with the half-life of each replicate. $\mathrm{p}$ : one-sided unpaired t-test.

(G) Flow cytometry analysis of strains expressing the UbiG76V-tFT reporter. mCherry/sfGFP ratios were normalized to the mean mCherry/sfGFP ratio of NAA10 wild type strains. AN: Rpn4A2N. p: one-sided unpaired t-test.

\section{Tom1 is an E4 ligase of the UFD}

Rpn4-independent destabilization of the UbiG76V-tFT reporter in ubr1 $\Delta$ ufd4 $\Delta$ cells upon deletion of NatA (Fig $2 G$ ) is consistent with our initial hypothesis, the existence of an unknown E3 ligase targeting this reporter for degradation. In human cells, the E3 ligase HUWE1 was implicated in the UFD pathway (Poulsen et al., 2012). The yeast homolog Tom1 targets excess histones (Singh et al., 2012), ribosomal subunits (Sung et al., 2016) and other proteins (Kim and Koepp, 2012; Kim et al., 2012) for degradation, but has not yet been described to mediate UFD. We used flow cytometry to test if Tom1 participates in degradation of UFD substrates and observed only weak stabilization of the UbiG76V-tFT reporter in the tom $1 \Delta$ mutant (Fig $3 A$, Pos. 1\&2). This could explain why Tom1 was not previously identified as a component of the UFD pathway. Nevertheless, we were able to co-immunoprecipitate C-terminally TAPtagged Tom1 with the UbiG76V-tFT reporter (Fig 3B), suggesting a direct role for Tom1 in degradation of UFD substrates.

According to the current model of the UFD pathway, linear ubiquitin fusions are first oligoubiquitinated by Ufd4 on the K29 residue of the N-terminal ubiquitin moiety (Johnson et al., 1995; Tsuchiya et al., 2013). These short chains are then extended by the chain-elongating E4 enzymes Ufd2 and Ubr1 to degradation-promoting length (Hwang et al., 2010a; Koegl et al., 1999). While Ubr1 activity has not been investigated in detail, Ufd2 is known to require K48 of the N-terminal ubiquitin moiety (Johnson et al., 1995; Koegl et al., 1999; Liu et al., 2017). The weak stabilization of the UbiG76V-tFT reporter in the tom $1 \Delta$ mutant suggests that Tom1 is redundant with Ufd4 or one of the E4 ligases. UFD substrates lacking K29 are fully stable (Johnson et al., 1995) and thus cannot be used to distinguish between these possibilities. To more confidently place Tom 1 in the UFD pathway, we therefore mutated K48 of the UbiG76VtFT reporter to arginine and measured turnover of the resulting UbiK48R,G76V-tFT reporter using flow cytometry. In wild type yeast, the UbiK48R, G76V-tFT reporter was degraded slower 
than the UbiG76V-tFT reporter and was not stabilized in a ufd2 $\Delta$ mutant, consistent with the current model. Strikingly, deletion of TOM1 almost completely abolished degradation of the UbiK48R,G76V-tFT reporter (Fig 3A, Pos. 8\&9) and the tom $1 \Delta$ and $u b r 1 \Delta$ ufd $4 \Delta$ mutants were indistinguishable in terms of UbiK48R,G76V-tFT turnover (Fig 3A, Pos. 9\&10). Interestingly, the UbiK48R,G76V-tFT reporter was slightly more stable in a tom $1 \Delta$ ubr1 $\Delta$ ufd $4 \Delta$ mutant compared to either tom $1 \Delta$ or $u b r 1 \Delta$ ufd $4 \Delta$ cells (Fig $3 A$, Pos. 9 to 11 ). Altogether, these observations argue that Tom1 can play a major role in degradation of UFD substrates. However, Tom1 is not essential for degradation of UFD substrates, as other ubiquitin ligases can use K48 to promote degradation of UFD substrates independently of Tom1. One such ligase is Ufd2, but it is likely that additional ligases performing this function exist, as the UbiG76V-tFT reporter was still degraded in a tom1 $\Delta$ ufd2 $\Delta$ mutant (Fig $3 \mathrm{~A}$ ).

\section{A}

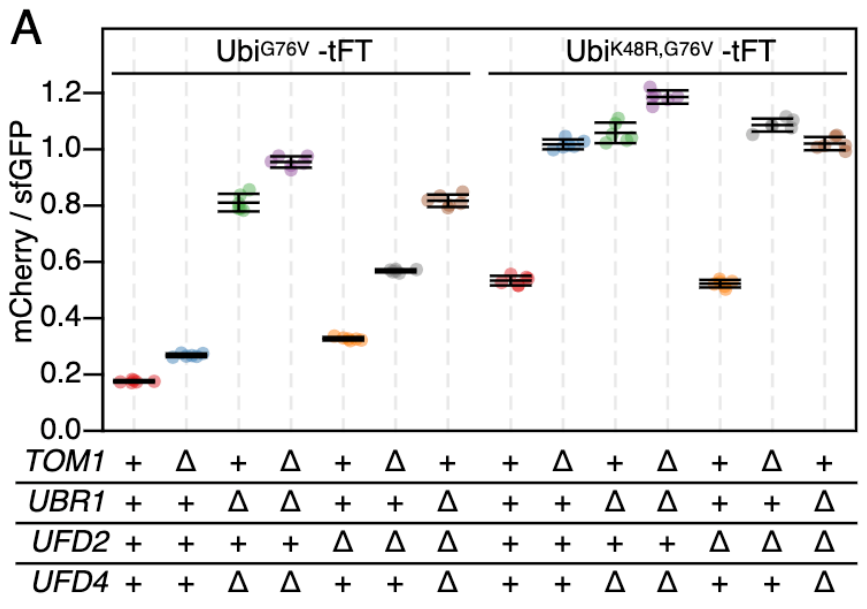

B

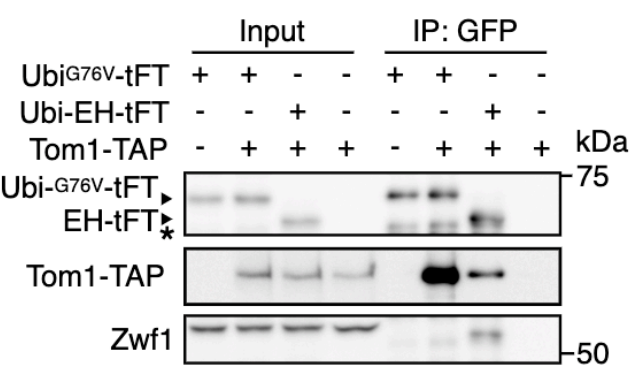

Fig 3. Role of Tom1 in degradation of UFD substrates.
(A) Flow cytometry analysis of strains expressing UbiG76V-tFT or UbiK48R,G76V-tFT reporters.

(B) Co-purification of Tom1 with the UbiG76V-tFT reporter. Proteins were separated by SDSPAGE followed by immunoblotting with antibodies against GFP, protein A, and Zwf1 as loading control. Input: whole-cell extract prepared by glass bead lysis. IP: proteins eluted after incubation of whole-cell extracts with GFP binding protein coupled to sepharose beads. The $\mathrm{EH}$ reporter is not a UFD substrate. It is therefore thought to not be targeted by Tom 1 and served as negative control. (*) marks a non-specific band.

We considered two mechanisms that could explain our results: (i) UFD substrates are ubiquitinated sequentially by Ufd 4 and Tom 1 and ubiquitination by Tom 1 depends on Ufd4; or (ii) Tom1 ubiquitinates UFD substrates independently of Ufd4 on a lysine residue distinct from K48. In the absence of E4 activity on K48, ubiquitination by either Ufd4 or Tom1 alone is not sufficient to target the reporter for degradation and both E3 ligases are required. To distinguish between these possibilities and to investigate the effect of Tom1 on ubiquitin chain formation, we purified ubiquitin conjugates from whole-cell extracts. The abundance of high molecular weight species originating from the UbiG76V-tFT reporter was reduced in the tom $1 \Delta$ mutant (Fig 4A, lanes 9\&13). Moreover, only mono- and diubiquitinated species were seen in the tom1 $\triangle$ mutant, when using the UbiK48R,G76V-tFT reporter, despite strong polyubiquitination 
of this reporter in wild type cells (Fig $4 \mathrm{~B}$, lanes $5 \& 7$ ). In a $u b r 1 \Delta$ ufd $4 \Delta$ background, the UbiK48R,G76V-tFT reporter was only weakly ubiquitinated (Fig 4B, lane 6). Altogether, these results are consistent with the idea that Tom1 acts as a chain elongating enzyme (E4) in the UFD pathway, which recognizes proteins that carry linear oligoubiquitin chains added by Ufd4 and extends these to a degradation-promoting length.

To test this hypothesis directly, we reconstituted ubiquitination of UFD substrates in vitro (Hwang et al., 2010a; Koegl et al., 1999). We first investigated ubiquitination by Ufd4, Ufd2, and Ubr1. Using Ubi-ProtA as a substrate, Ubr1 or Ufd4 alone generated short ubiquitin chains of up to three or four ubiquitin monomers in length, respectively, while Ufd2 was inactive in the absence of other E3 ligases (Fig 4C, lanes 1 to 5). On the other hand, Ufd4 combined with Ufd2 and/or Ubr1 generated high molecular weight conjugates (Fig 4C, lanes 6 to 8). When UbiK48R-ProtA was used as a substrate, the combination of Ufd4 and Ufd2 did not synthesize appreciable amounts of polyubiquitin conjugates (Fig 4D, lanes $3 \& 7$ ). Altogether, these results reproduce previous observations (Hwang et al., 2010a; Koegl et al., 1999) and hence confirm the integrity of our in vitro system. Next, we used this assay to investigate the effect of Tom1 on ubiquitin chain formation. Tom1 alone was inactive towards both Ubi-ProtA and UbiK48RProtA, but generated high molecular weight polyubiquitin chains in the presence of Ufd4 regardless of the model substrate (Fig $4 C$ and $D$, lanes 1, 3, 9 \& 11 each). This indicates that Tom1 recognizes oligoubiquitinated UFD substrates and either extends pre-formed chains or synthesizes new chains conjugated directly to the substrate, but using a residue distinct from K48 of the N-terminal ubiquitin moiety for chain attachment. Since HUWE1, the mammalian homologue, was shown to synthesize K6- and K11-linked chains (Michel et al., 2017; Yau et al., 2017), it is possible that Tom1 can use those lysine residues of the $\mathrm{N}$-terminal ubiquitin moiety to initiate new chains. Moreover, detailed analysis of the banding pattern revealed that in the presence of Tom 1 tri-ubiquitinated species of different apparent molecular weight were generated (Fig 4E), indicating that ubiquitin conjugates synthesized by Tom1 and Ufd4 are clearly distinct.

We next used mass spectroscopy in order to identify the type of linkages formed in the in vitro ubiquitination reactions. In reactions that included Ufd4 alone (Fig 4C, lane 3), only K29 linkages were observed (Fig 4F) as expected (Koegl et al., 1999; Liu et al., 2017). Upon addition of Tom1 a strong signal for K48 linkages was observed (Fig 4F) indicating the formation of elongated chains based on K48 linkages. When we tested the high molecular weight products of full reactions (Fig. 4C, lane 15) that included Ufd4, Ufd2 and Tom1 we could also detect $\mathrm{K} 11$ linkages, whereas these linkages were absent in this fraction when Tom1 was omitted (Fig. 4C, lane 8). Together these results support the idea Tom1 functions as an E4 enzyme and that it is able to form different types of ubiquitin linkages. 
A

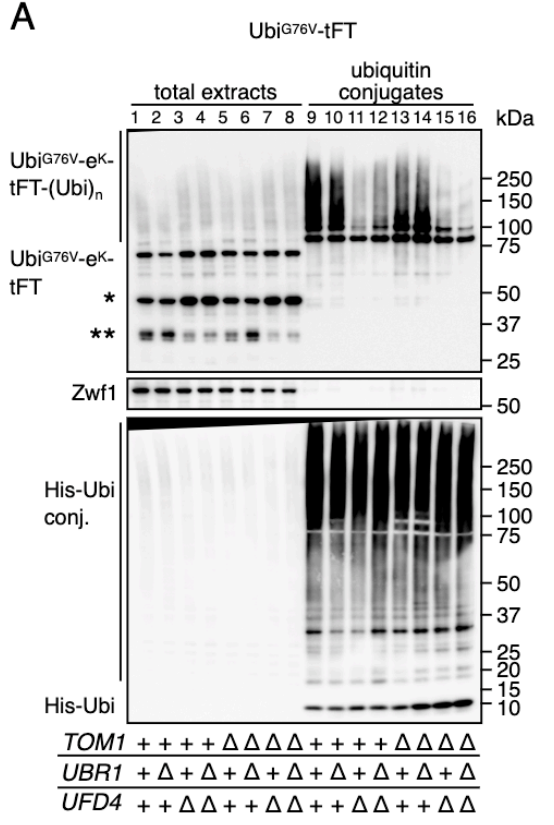

B

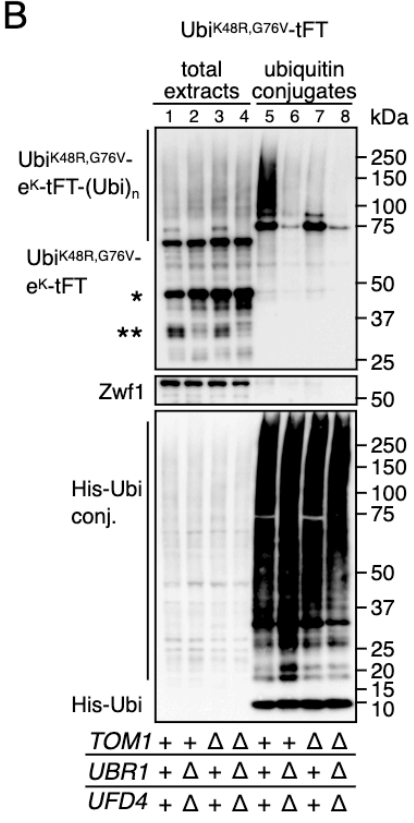

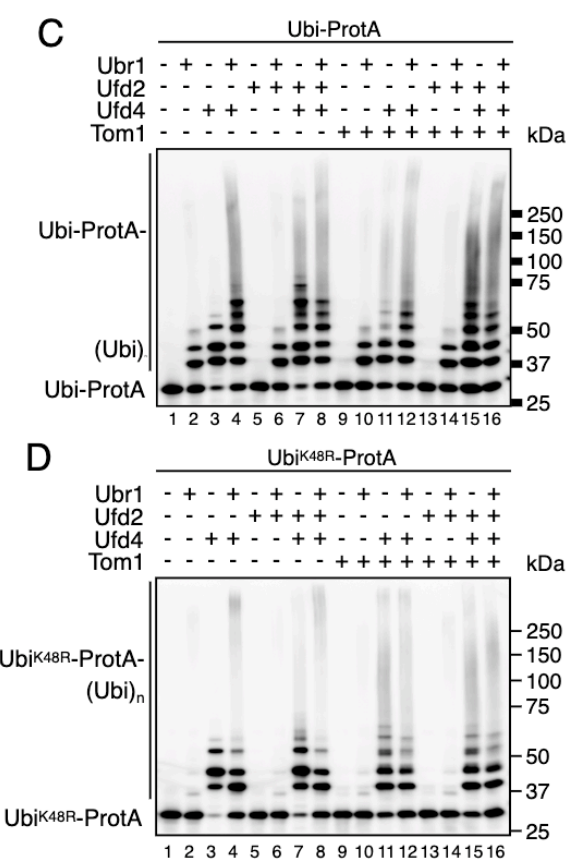

$\mathrm{E}$

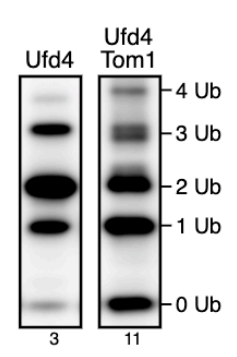

$\mathrm{F}$

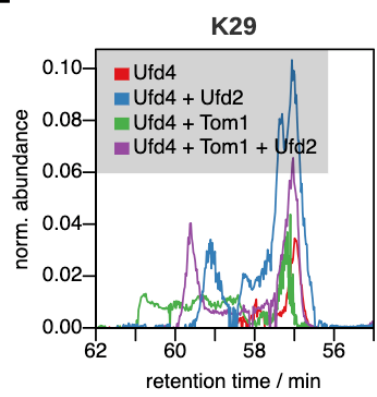

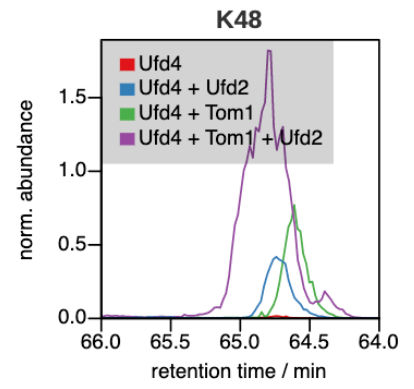

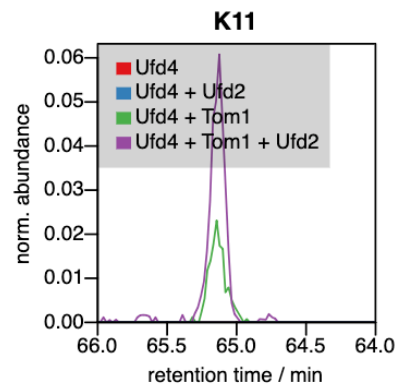

Fig 4. Tom1 is an E4 ubiquitin ligase and catalyzes the formation of K48 and K11 ubiquitin linkages

(A and B) Ubiquitination of UbiG76V-tFT (A) or UbiK48R,G76V-tFT (B) in strains expressing 10xHis-tagged ubiquitin. Total cell extracts and ubiquitin conjugates purified by immobilized metal affinity chromatography were analyzed by SDS-PAGE followed by immunoblotting against GFP, Zwf1, and ubiquitin. A product of mCherry hydrolysis during cell extract preparation (Gross et al., 2000) $(*)$ and a product resulting from inefficient proteasomal degradation of sfGFP (Khmelinskii et al., 2016) $(* *)$ are marked.

(C and D) In vitro reconstitution of ubiquitin chain formation with Ubi-ProtA (C) or UbiK48RProtA (D) as substrate using immunoblotting against protein $A$.

(E) Comparison of the banding pattern of lanes 3 and 11 from (D). Length of ubiquitin chains is indicated.

(F) Analysis of ubiquitin linkages by mass spec. Ubiquitinated proteins were isolated from SDS PAGE gels prepared from samples in (C) and analyzed for the presence of branched chains as described in methods. The abundance of characteristic fragments in the eluates is shown. Traces were normalized to the non-modified K63 peptide.

Next, we tested if Tom1 contributes to the destabilization of UFD substrates in NatA-deficient cells. In the ubr1 $\Delta$ ufd $4 \Delta$ background, cells lacking Tom1 showed a markedly reduced acceleration of UbiG76V-tFT reporter degradation upon deletion of NAA10 compared to Tom1- 
proficient cells, and this destabilization was further reduced, but not completely abolished, in cells carrying the rpn4A2N allele (Fig 5A). These results indicate that accelerated turnover of UFD substrates in the naa $10 \Delta$ mutant is mediated partially by Tom1, partially by reduced ubiquitin-independent degradation of Rpn4, and partially by other factors.

Increased abundance of Tom1 and/or other UFD-specific E3 ligases in the naa10 background could explain accelerated turnover of UFD substrates in this mutant. Supporting this notion, elevated levels of Ubr1 in NatA-deficient cells have been observed previously (Oh et al., 2017). We therefore tested if NatA affects abundance of Ufd4 and Tom1 using immunoblotting. Levels of both E3 ligases were elevated in naa10 $\Delta$ cells compared to wild type (Fig 5A and Fig S3).

To test if increased abundance of E3s participating in UFD can accelerate degradation of UFD substrates, we measured degradation of UbiG76V-tFT and Ubi-PZ-tFT reporters in strains overexpressing Ufd4, Tom1, or Ubr1 using flow cytometry. No clear changes in turnover of the UbiG76V reporter were detected, most likely because it is at the lower limit of the tFT dynamic range in wild type cells. The Ubi-PP-tFT reporter was more stable in the wild type background but was only weakly destabilized in a strain overexpressing Ubr1 (Fig 5B), consistent with a negligible contribution of Ubr1 to UFD in vivo (Figs 1C and 3C (Hwang et al., 2010a)). However, overexpression of Ufd4 or Tom1 strongly destabilized the PP reporter (Fig 5B). Only a fraction of this reporter is degraded by the UFD pathway, while the other fraction is stable due to removal of the N-terminal ubiquitin moiety by DUBs. Increased turnover of this reporter upon overexpression of Ufd4 and Tom1 therefore indicates that these E3 ligases can compete with DUB activity. Moreover, these results suggest that increased abundance of UFD E3 ligases could explain accelerated turnover of UFD substrates in the naa10 $\Delta$ mutant.

Given that deletion of NAA10 in a ubr1 $\Delta$ ufd $4 \Delta$ tom $1 \Delta$ rpn $4 A 2 N$ background still slightly accelerated degradation of the UbiG76V-tFT reporter (Fig $5 \mathrm{~A}$ ), we hypothesize that this destabilization is not due to the action of one single protein, but rather the result of a systemic upregulation of the UPS, caused in part by reduced ubiquitin-independent degradation of Rpn4, but also by other factors currently unknown. A reason for this could be unspecific, lowefficiency ubiquitination of the $\mathrm{N}$-terminal ubiquitin moiety by most, if not all, cellular E3 ligases, in addition to the specific, high-efficiency ubiquitination by Ufd4 and Tom1. Upregulation of the UPS would therefore lead to not only an increase in specific and unspecific ubiquitination of UFD substrates but also accelerated proteasomal degradation.

A

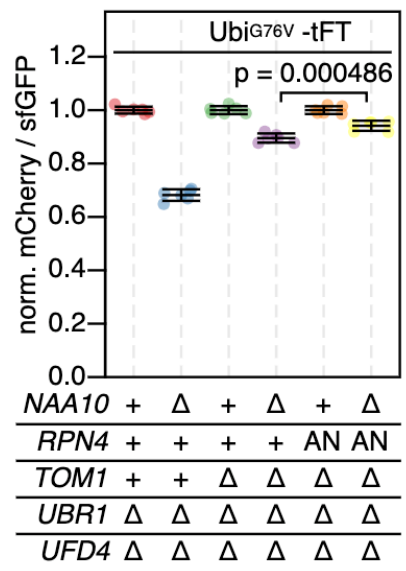

B

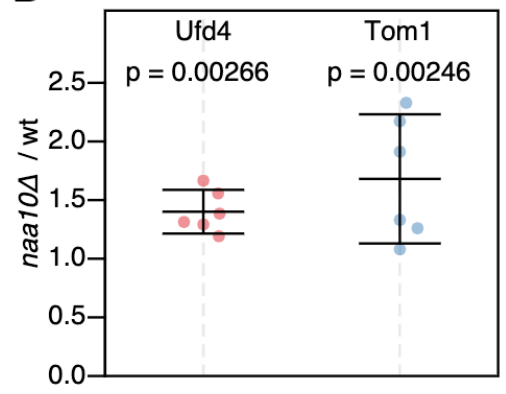

C

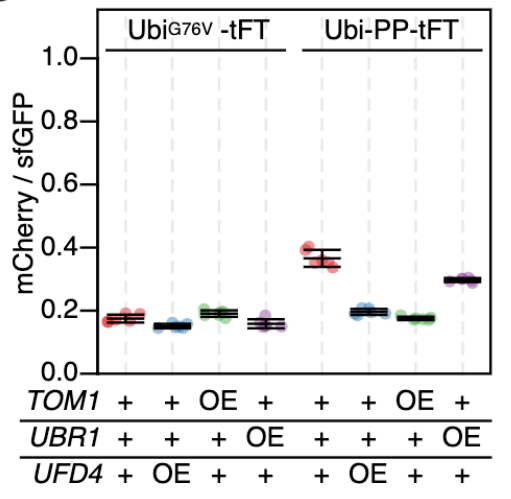

Fig 5. Role of NatA in regulation of the UFD. 
(A) Flow cytometry analysis of strains expressing the UbiG76V-tFT reporter. mCherry/sfGFP ratios were normalized to the mean mCherry/sfGFP ratio of NAA10 wild type strains. AN: Rpn4A2N. p: one-sided unpaired t-test.

(B) Abundance of C-terminally 3HA-tagged Ufd4 or TAP-tagged Tom1 in cells lacking NatA compared to wild type yeast. Whole-cell extracts were separated by SDS-PAGE followed by immunoblotting with antibodies against HA and Pgk1 (Ufd4) or with antibodies against protein $\mathrm{A}$ and Fas (Tom1). Pgk1 and Fas served as loading controls. Mean fold-change and $95 \% \mathrm{Cl}$ of six replicates are plotted together with the fold-change of each replicate. $p$ : one-sample $t-$ test.

(C) Flow cytometry analysis of strains expressing UbiG76V-tFT or Ubi-PP-tFT reporters. OE: overexpression from the GPD promoter.

\section{Discussion}

Our study sheds light on the impact of NatA Nt-acetylation on protein homeostasis. NatA mutants exhibit specific phenotypes, some of which can be explained by impaired proteinprotein interactions in the absence of correctly acetylated $\mathrm{N}$-termini, with various consequences: transcriptional alterations caused by defective Sir3-dependent gene silencing (Wang et al.), impaired function and stability of the Hsp90 chaperonin system and its client proteins (Oh:2017hx), cellular sorting of secretory proteins, functions of the Golgi apparatus and the actin cytoskeleton and targeting of specific proteins for degradation (summarized in (Aksnes et al.)). It is easy to imagine that a multitude of individual effects can challenge proteostasis regulation that then demands for a higher activity of the UPS in order to remove damage: mistargeted proteins, misfolded proteins, mis-expressed proteins and subunits. This higher UPS activity then could at least partially account for the increased degradation rate of linear ubiquitin fusion proteins.

Interestingly, our observation that Rpn4 Nt-acetylation enhances the strength of its Nt-degron provides a hint towards a more direct coupling of NatA and proteostasis regulation. Here we demonstrate that Nt-acetylation can act independently of E3 ligases to promote ubiquitinindependent degradation of Rpn4, thereby linking NatA activity to regulation of UPS activity. Importantly, in this context Nt-acetylation is neither required nor sufficient to trigger degradation of Rpn4, but rather accelerates degradation of this already unstable protein. Although abundance and half-life of Rpn4 were increased in NatA-deficient cells, we did not observe clearly increased activity of proteasomal subunit promoters (S4 Fig). This could be explained by the relatively weak effect of NatA on Rpn4 degradation and abundance, and it is consistent with the previous report that the abundance of proteasomal subunits was not significantly increased even when the N-terminal degron of Rpn4 was completely removed (Wang et al., 2004a), and that it showed only a modest increase in response to expression of a nondegradable Rpn4 variant lacking both degrons (Wang et al., 2010). Since promoters of E3 ligases involved in UFD appear to lack obvious Rpn4 binding motifs (Shirozu et al., 2015) it is unlikely that the increased abundance of Tom1, Ubr4 (Fig. 5b) and Ubr1 (Oh et al., 2017) E3 ligases in the naa10 $\Delta$ mutant is mediated by Rpn4. It can be imagined that load-dependent inhibition of autoubiquitination regulates E3 abundance, as shown for other E3 ligases ( $P$ de Bie, 2011). Alternatively, Rpn4-independent NatA-mediated regulation of E3 expression is possible.

We furthermore show that degradation of UFD substrates is accelerated in NatA-deficient cells and subsequently identify the E3 ligase Tom1 as a novel E4 chain elongating enzyme of this 
pathway. This function of Tom1 is clearly distinct from its previously recognized roles as an E3 ligase that is sufficient for ubiquitination of substrate proteins (Sung et al., 2016) and its E3independent function in ribosome-associated quality control (Defenouillère et al., 2013). While no endogenous substrates of the UFD pathway are known in yeast, the pathway is conserved in mammalian cells, where several functions have been identified. UBB+1, a mutant ubiquitin variant with a short C-terminal extension caused by a frameshift mutation, is a substrate of the mammalian UFD pathway (Park et al., 2009) and has been linked to neurodegenerative disorders (van Leeuwen et al., 1998). The cell cycle regulator p21 (Bloom et al., 2003), the ERK3 MAP kinase (Coulombe et al., 2004), and the Arf tumor suppressor (Kuo et al., 2004) were shown to be degraded following $\mathrm{N}$-terminal ubiquitination. It was recently demonstrated that HUWE1, the mammalian ortholog of Tom1, can ubiquitinate MyoD, the first known UFD substrate, on its N-terminal residue (Noy et al., 2012). Given the conserved nature of UFD and its components, we speculate that Tom1 can generate endogenous UFD substrates in yeast. Altogether, our results complement the knowledge about the role of NatA dependent Ntterminal acetylation and how this is coupled to the activity of the UPS. We believe that our work will contribute to a better understanding of this protein modification and its functions.

\section{Materials and Methods}

\section{Yeast genome manipulations}

Yeast gene deletions and promoter duplications were performed by PCR targeting, as described (Huber et al., 2014; Janke et al., 2004). Seamless genome editing was performed using the 50:50 technique (Horecka and Davis, 2014). Yeast strains and plasmids used in this study are listed in Tables S1 and S2, respectively.

\section{tFT-based protein stability measurements with flow cytometry (tFT assay)}

Yeast strains containing the desired plasmids were inoculated into $200 \mu \mathrm{l} \mathrm{SC}$ medium lacking the appropriate amino acids for plasmid selection and grown to saturation in 96-well plates. The cultures were then diluted into fresh medium by pinning to a new 96-well plate using a RoToR pinning robot (Singer Instruments) and incubated at $23^{\circ} \mathrm{C}$ for $20-24 \mathrm{~h}$ to $1 \times 10^{6}-8 \times 10^{6}$ cells/ml. Flow cytometry was performed on a FACSCanto RUO HTS flow cytometer (BD Biosciences) equipped with a high-throughput sample loader, a $561 \mathrm{~nm}$ laser with $600 \mathrm{~nm}$ long pass and $610 / 20 \mathrm{~nm}$ band pass filters for mCherry, and a $488 \mathrm{~nm}$ laser with $505 \mathrm{~nm}$ long pass and $530 / 30 \mathrm{~nm}$ band pass filters for sfGFP. Data analysis was performed in $R$ (R Core Team, 2016) with the flowCore and flowWorkspace packages using a custom script. Briefly, the events were gated for mCherry- and sfGFP-positive cells, the median intensity of a negative control was subtracted from each channel, and the mCherry/sfGFP ratio was calculated for each cell. The median mCherry/sfGFP ratio of each sample was used for further analysis. Unless otherwise stated, each experiment was performed using two biological replicates with three technical replicates each. To account for growth rate differences, sample mCherry/sfGFP ratios were normalized to the stable Ubi-TH-eK-tFT reporter (plasmid pAnB19-TH, Table S2), which was measured in each strain background.

\section{Flow cytometry of promoter duplications}

Yeast cells were inoculated into $200 \mu \mathrm{l} \mathrm{SC}$ medium and grown to saturation in 96-well plates. The cultures were then diluted into fresh medium by pinning to a new 96 -well plate using a 
RoToR pinning robot (Singer Instruments) and incubated at $23^{\circ} \mathrm{C}$ for $20-24 \mathrm{~h}$ to $1 \times 10^{6}-8 \times 10^{6}$ cells/ml. Flow cytometry was performed on a FACSCanto RUO HTS flow cytometer (BD Biosciences) equipped with a high-throughput sample loader, a $561 \mathrm{~nm}$ laser with $600 \mathrm{~nm}$ long pass and 610/20 nm band pass filters for mCherry, and a $488 \mathrm{~nm}$ laser with $505 \mathrm{~nm}$ long pass and 530/30 $\mathrm{nm}$ band pass filters for sfGFP. Data analysis was performed in R (R Core Team, 2016) with the flowCore and flowWorkspace packages using a custom script. Briefly, the events were gated for single cells using forward and side scatter pulse width, followed by gating for fluorescent cells. The median intensity of a negative control was subtracted from each cell. The median sfGFP intensity of each sample was used for further analysis. Unless otherwise stated, each experiment was performed using two biological replicates with three technical replicates each.

\section{Cycloheximide chases}

Cells were grown at $23^{\circ} \mathrm{C}$ to $6 \times 10^{6}-1 \times 10^{7}$ cells $/ \mathrm{ml}$ in synthetic medium before addition of cycloheximide (Sigma Aldrich, $100 \mathrm{mg} / \mathrm{ml}$ stock in $100 \%$ ethanol) to $100 \mu \mathrm{g} / \mathrm{ml}$ final concentration. At each time point, $1 \mathrm{ml}$ of the culture was removed, mixed with $150 \mu \mathrm{l} 1.85 \mathrm{M}$ $\mathrm{NaOH}$ and $10 \mu \mathrm{l}$ 2-mercaptoethanol and flash-frozen in liquid nitrogen. Protein extracts were prepared as described (Knop et al., 1999), followed by SDS-PAGE and immunoblotting.

For Ubi-P-tFT constructs, membranes were probed with rabbit anti-GFP (ab6556, Abcam) and mouse anti-Pgk1 (22C5D8, Molecular Probes) antibodies. A secondary donkey anti-mouse antibody coupled to IRDye800 (610-732-002, biomol, Rockland) or donkey anti-rabbit coupled to Alexa 680 (A10043, life technologies) were used for detection on an Odyssey infrared imaging system (Li-Cor).

For Rpn4-TAP strains, membranes were probed with rabbit peroxidase-anti-peroxidase (PAP) antibodies (Z0113, Dako) and imaged on an LAS-4000 system (Fuji), followed by probing with mouse anti-Pgk1 (22C5D8, Molecular Probes) and goat anti-mouse HRP (115-035-003, Dianova) antibodies and imaging. Quantification was performed in ImageJ (Schneider et al., 2012).

For HA-tagged Ufd4, membranes were probed with mouse anti-HA (12CA5) and mouse antiPgk1 (22C5D8, Molecular Probes), followed by probing with mouse anti-Pgk1 (22C5D8, Molecular Probes) and imaging on a LAS-4000 system (Fuji).

\section{Tom1 abundance}

Cells expressing protein A-tagged Tom 1 were grown at $23^{\circ} \mathrm{C}$ to $6 \times 10^{6}-1 \times 10^{7} \mathrm{cells} / \mathrm{ml}$ in synthetic medium and samples were taken and cell extracts were prepared as described (Knop et al., 1999). Following SDS PAGE and Western blotting, membranes were probed with rabbit peroxidase-anti-peroxidase (PAP) antibodies (Z0113, Dako) and imaged on an LAS-4000 system (Fuji), followed by probing with rabbit anti-Fas (Egner et al., 1993) and goat anti-rabbit HRP (111-035-003, Dianova) antibodies and imaging. Quantification was performed in ImageJ (Schneider et al., 2012).

\section{Rpn4 mass spectrometry}

pdr5 $\Delta$ ubr2 $\Delta$ yeast cells expressing transcriptionally inactive Rpn4C477A mutants (Wang et al, 2004) C-terminally tagged with 10xHis-sfGFPcp8 (Khmelinskii et al., 2016)from a GPD 
promoter were grown in SC-His to $7 \times 10^{6}-8 \times 10^{6} \mathrm{cells} / \mathrm{ml}$. Bortezomib was added to $50 \mu \mathrm{M}$ and cultures were incubated for $1 \mathrm{~h} .2 .5 \times 10^{9}$ cells were harvested by centrifugation, washed with $20 \%(\mathrm{w} / \mathrm{v})$ trichloroacetic acid, and stored at $-80^{\circ} \mathrm{C}$. Cell pellets were resuspended in 1600 $\mu \mathrm{l} 20 \%$ (w/v) trichloroacetic acid and lysed with $0.5 \mathrm{~mm}$ glass beads (Sigma) in a FastPrep FP120 (Thermo) for $8 \times 40 \mathrm{~s}$ at $6.5 \mathrm{~m} / \mathrm{s}$. After precipitation, proteins were washed with cold acetone, air-dried and resuspended in $3 \mathrm{ml}$ purification buffer (6M guanidium chloride, $100 \mathrm{mM}$ Tris- $\mathrm{HCl} \mathrm{pH} \mathrm{9.0,} 300 \mathrm{mM} \mathrm{NaCl}, 10 \mathrm{mM}$ imidazole, $0.2 \%$ (v/v) Triton X-100). DTT was added to $10 \mathrm{mM}$ and samples were incubated at $60^{\circ} \mathrm{C}$ for $30 \mathrm{~min}$, followed by quenching with 100 $\mathrm{mM}$ chloroacetamide at RT for $60 \mathrm{~min}$. Lysates were clarified at $21000 \mathrm{~g}, 4^{\circ} \mathrm{C}$ for $45 \mathrm{~min}$ and the supernatants incubated with TALON beads (Clontech) pre-equlibrated with purification buffer at RT over night with overhead rotation followed by washing with wash buffer (8M urea, $100 \mathrm{mM}$ sodium phosphate $\mathrm{pH} 7.0,300 \mathrm{mM} \mathrm{NaCl}, 5 \mathrm{mM}$ imidazole, $5 \mathrm{mM}$ chloroacetamide, $0.2 \%(\mathrm{v} / \mathrm{v})$ Triton X-100) without (twice) and with $0.2 \%(\mathrm{w} / \mathrm{v})$ SDS (twice). Rpn4 was eluted in $30 \mu \mathrm{l}$ elution buffer (8M urea, $100 \mathrm{mM}$ sodium phosphate $\mathrm{pH} 7.0,300 \mathrm{mM} \mathrm{NaCl}, 500 \mathrm{mM}$ imidazole, $5 \mathrm{mM}$ chloroacetamide, $0.2 \%$ (v/v) Triton X-100). $7 \mu \mathrm{l}$ of eluate were used for SDSPAGE followed by Coomassie Brilliant Blue staining. Bands of the expected size were excised, digested with trypsin, and analyzed with ESI LC-MS/MS on a Q-Exactive HF (Thermo Scientific) coupled with Dionex Ultimate 3000 RSLCnano (Thermo Scientific). Mass spectrometry was performed at the ZMBH core facility for mass spectrometry and proteomics.

\section{Ubiquitin pulldowns}

Ubiquitinated proteins were purified from yeast cells expressing $\mathrm{N}$-terminally 10xHis-tagged ubiquitin using a protocol adapted from (Khmelinskii et al., 2014). Yeast were grown in SCHis/Leu to $7 \times 10^{6}-8 \times 10^{6}$ cells/ml. Approx. $1 \times 10^{9}$ cells were harvested by centrifugation, washed with cold $\mathrm{H} \neg 2 \mathrm{O}$, and stored at $-80{ }^{\circ} \mathrm{C}$. Cell pellets were resuspended in $800 \mu \mathrm{l} 20 \%$ $(\mathrm{w} / \mathrm{v})$ trichloroacetic acid and lysed with $0.5 \mathrm{~mm}$ glass beads (Sigma) in a FastPrep FP120 (Thermo) for $8 \times 40 \mathrm{~s}$ at $6.5 \mathrm{~m} / \mathrm{s}$. After precipitation, proteins were washed with cold acetone, air-dried, resuspended in $1.5 \mathrm{ml}$ purification buffer $(6 \mathrm{M}$ guanidium chloride, $100 \mathrm{mM}$ Tris- $\mathrm{HCl}$ $\mathrm{pH}$ 9.0, $300 \mathrm{mM} \mathrm{NaCl}, 10 \mathrm{mM}$ imidazole, $5 \mathrm{mM}$ chloroacetamide, $0.2 \%$ (v/v) Triton X-100), and clarified at $21000 \mathrm{~g}, 4{ }^{\circ} \mathrm{C}$ for $45 \mathrm{~min}$. Protein concentration was determined with Bradford assay (BioRad) in purification buffer diluted $1: 10$ with $\mathrm{H}_{2} \mathrm{O}$. $1 \%$ of the amount of protein to be used for purification was removed, precipitated with $150 \mu \mathrm{l} 20 \%(\mathrm{w} / \mathrm{v})$ trichloroacetic acid, and resuspended in $100 \mu \mathrm{HU}$ buffer (8 M Urea, 5\% (w/v) SDS, $200 \mathrm{mM}$ sodium phosphate $\mathrm{pH}$ 7.0, $1 \mathrm{mM}$ EDTA, $15 \mathrm{mg} / \mathrm{ml}$ DTT) to be used as total extract. Equal amounts of protein were incubated with TALON beads (Clontech) pre-equilibrated with purification buffer at RT for 1.5 $\mathrm{h}$ with overhead rotation, followed by washing with wash buffer (8M urea, $100 \mathrm{mM}$ sodium phosphate $\mathrm{pH} 7.0,300 \mathrm{mM} \mathrm{NaCl}, 5 \mathrm{mM}$ imidazole, $5 \mathrm{mM}$ chloroacetamide, $0.2 \%(\mathrm{v} / \mathrm{v})$ Triton $\mathrm{X}-100$ ) without (twice) and with $0.2 \%(\mathrm{w} / \mathrm{v})$ SDS (twice). Ubiquitin conjugates were eluted in 50 $\mu \mathrm{l}$ elution buffer (8M urea, $100 \mathrm{mM}$ sodium phosphate $\mathrm{pH} 7.0,300 \mathrm{mM} \mathrm{NaCl}, 500 \mathrm{mM}$ imidazole, $5 \mathrm{mM}$ chloroacetamide, $0.2 \%(\mathrm{v} / \mathrm{v})$ Triton X-100) and analyzed by SDS-PAGE on 4-12\% NuPAGE Bis-Tris gradient gels (Invitrogen) followed by immunoblotting. After probing with rabbit anti-GFP (ab6556, Abcam) and rabbit anti-Zwf1 (Miller et al., 2015) followed by goat anti-rabbit IgG-HRP (\#111-035-003, Dianova) and imaging on an LAS-4000 system (Fuji), membranes were stripped (100 mM glycine, $2 \%(\mathrm{w} / \mathrm{v}) \mathrm{SDS}, \mathrm{pH} 2.0)$ and re-probed with mouse anti-ubiquitin (P4G7) followed by goat anti-mouse IgG-HRP (\#115-035-003, Dianova) and imaging. 


\section{LC-MS Analysis of ubiquitin linkages}

SDS-PAGE gels of in vitro ubiquitination products (Fig. 4C,D) were stained using Coomassie and from each lane the regions corresponding to the polyubiquitinated species were cut out and processed as described with minor modifications (Fecher-Trost et al., 2013). In brief, after reduction with dithiothreitol and alkylation with iodoacetamide, trypsin digestion was done overnight at $37^{\circ} \mathrm{C}$. The reaction was quenched by addition of $20 \mu \mathrm{L}$ of $0.1 \%$ trifluoroacetic acid (TFA; Biosolve, Valkenswaard, The Netherlands) and the supernatant was dried in a vacuum concentrator before LC-MS analysis. Nanoflow LC-MS2 analysis was performed with an Ultimate 3000 liquid chromatography system coupled to an QExactive HF mass spectrometer (Thermo-Fischer, Bremen, Germany). Samples were dissolved in 0.1\% TFA and injected to a self-packed analytical column (75um x 200mm; ReproSil Pur 120 C18-AQ; Dr Maisch GmbH) and eluted with a flow rate of $300 \mathrm{nl} / \mathrm{min}$ in an acetonitrile-gradient (3\%-40\%). The mass spectrometer was operated in data-dependent acquisition mode, automatically switching between MS and MS2. Collision induced dissociation MS2 spectra were generated for up to 20 precursors with normalized collision energy of $29 \%$.

Database search - Raw files were processed using Proteome Discoverer 2.3. (Thermo Scientific) for peptide identification and quantification. MS2 spectra were searched with the SEQUEST software (Thermo Scientific) against the Uniprot yeast database (6910 entries) and the contaminants database (MaxQuant version 1.5.3.30 (Cox and Mann, 2008) with the following parameters: Carbamidomethylation of cysteine residues as fixed modification and Acetyl (Protein N-term), Oxidation (M), deamidation (NQ) and GG signature for ubiquitination $(\mathrm{K})$ as variable modifications, trypsin/P as the proteolytic enzyme with up to 2 missed cleavages. Peptide identifications were accepted if they could be established at greater than $95,0 \%$ probability by the Peptide Prophet algorithm (Keller et al., 2002). Protein identifications were accepted if they could be established at greater than $95,0 \%$ probability and contained at least 2 identified peptides. Protein probabilities were assigned by the Protein Prophet algorithm (Alexey et al., 2003). Scaffold (version Scaffold_4.8.4, Proteome Software Inc., Portland, Oregon) was used to validate and visualize MS/MS based peptide and protein identifications. For graphic presentation of XICs retention times were aligned and exported as .csv files using FreeStyle (Thermo Scientific).

\section{Tom1 co-immunoprecipitation}

Yeast strains expressing the desired construct were grown to $7 \times 10^{6}-8 \times 10^{6}$ cells $/ \mathrm{ml} .1 \times 10^{9}$ were harvested by centrifugation, washed with cold $\mathrm{H} 2 \mathrm{O}$, and stored at $-80^{\circ} \mathrm{C}$. GFP fusions were immunoprecipitated using lab-purified GFP binding protein (GBP) (Rothbauer et al., 2008) coupled to NHS-activated Sepharose FastFlow beads (GE Healthcare) using a protocol adapted from (Babiano et al., 2012). Cell pellets were resuspended in $200 \mu \mathrm{l}$ cold lysis buffer (50 mM Tris-HCl pH 7.4, 150 mM CH3COOK, 5 mM EDTA, 5 mM EGTA, $0.2 \%$ Triton X-100) with protease inhibitors (2x Roche Complete EDTAfree, $5 \mathrm{mM}$ benzamidine, $5 \mathrm{mM}$ Pefabloc SC, $5 \mathrm{mM} \mathrm{1,10-phenanthroline,} 25 \mathrm{mM} \mathrm{N}$-ethylmaleimide) and lysed with $0.5 \mathrm{~mm}$ glass beads (Sigma) in a FastPrep FP120 for $6 \times 20 \mathrm{~s}$ at $6.5 \mathrm{~m} / \mathrm{s}$. Lysates were clarified at $21000 \mathrm{~g}$ for 30 min and the supernatants incubated for $2 \mathrm{~h}$ at $4{ }^{\circ} \mathrm{C}$ with overhead rotation together with $40 \mu \mathrm{l}$ GBP-beads previously equilibrated by washing 3 times with $1 \mathrm{ml}$ lysis buffer. The beads were washed 3 times with lysis buffer and eluted in $50 \mu \mathrm{HU}$ buffer (8 M Urea, 5\% (w/v) SDS, 200 mM sodium phosphate $\mathrm{pH}$ 7.0, $1 \mathrm{mM}$ EDTA, $15 \mathrm{mg} / \mathrm{ml}$ DTT). Samples were analyzed by SDSPAGE followed by immunoblotting with rabbit peroxidase-anti-peroxidase (PAP) antibodies (Z0113, Dako) or rabbit anti-GFP (ab6556, Abcam) and rabbit anti-Zwf1 (Miller et al., 2015) 
followed by goat anti-rabbit IgG-HRP (\#111-035-003, Dianova) and imaging on an LAS-4000 system (Fuji).

\section{In vitro ubiquitination assays}

6xHis-Rad6, 6xHis-Ubc4, Ubi-ProtA-6xHis, and UbiK48R-ProtA-6xHis were expressed in E.coli BL21(DE3) pRIL and purified over a pre-packed HisTrap FastFlow column (GE Healthcare). FLAG-Ufd4, FLAG-Ubr1, FLAG-Ufd2, and FLAG-Tom1 were overexpressed in yeast from a GPD promoter and purified as described (Hwang et al., 2009; Hwang and Varshavsky, 2008). Purified yeast Uba1 and ubiquitin were purchased from BostonBiochem (\#E-300 and \#U-100SC, respectively). Final protein concentrations were $100 \mathrm{nM}$ (Uba1), 80 $\mu \mathrm{M}$ (ubiquitin), $1 \mu \mathrm{M}$ (Rad6), $1 \mu \mathrm{M}$ (Ubc4), $200 \mathrm{nM}$ (Ubr1), $200 \mathrm{nM}$ (Ufd4), 200 nM (Ufd2), 200 $\mathrm{nM}$ (Tom1), $125 \mathrm{nM}$ (Ubi-ProtA), $125 \mathrm{nM}$ (UbiK48R-ProtA), in $20 \mu$ reactions containing $4 \mathrm{mM}$ ATP (\#1191, Merck), $150 \mathrm{mM} \mathrm{NaCl}, 5 \mathrm{mM} \mathrm{MgCl2,} 1 \mathrm{mM}$ DTT, $50 \mathrm{mM}$ HEPES (pH 7.5). All reactions contained Uba1, ubiquitin, Rad6, and Ubc4. Reactions were pipetted on ice, incubated at $30{ }^{\circ} \mathrm{C}$ for $30 \mathrm{~min}$, quenched by addition of $20 \mu \mathrm{l} 5 \mathrm{xDS}$ sample buffer $(50 \%(\mathrm{v} / \mathrm{v})$ glycerol, 10\% (w/v) SDS, $250 \mathrm{mM}$ Tris- $\mathrm{HCl} \mathrm{pH}$ 6.8, $62.5 \mathrm{mM}$ EDTA, 5\% (v/v) ßmercaptoethanol) and incubation at $95^{\circ} \mathrm{C}$ for $5 \mathrm{~min}$, and analyzed using 4-12\% NuPAGE BisTris gradient gels (Invitrogen) followed by immunoblotting with rabbit peroxidase-antiperoxidase (PAP) antibodies (Z0113, Dako) and imaging on a LAS-4000 system (Fuji).

\section{Fluorescence microscopy}

Yeast strains were grown in SC medium at $23^{\circ} \mathrm{C}$ to $\sim 8 \times 10^{6}$ cells $/ \mathrm{ml}$. Control strains not expressing fluorescent proteins and Tom1-GFP strains additionally expressing mCherry from a constitutive promoter were mixed 1:1 and attached to glass-bottom 96-well plates (MGB0961-2-LG-L, Matrical) as described (Khmelinskii and Knop, 2014). Image stacks were acquired on a Nikon Ti-E wide field epifluorescence microscope with a 60x ApoTIRF oil immersion objective (1.49 NA, Nikon), an LED light source (SpectraX, Lumencor), a Flash4 sCMOS camera (Hamamatsu). Segmentation was performed in the bright-field channel using CellX (Mayer et al., 2013). Flat-field correction was performed using a reference image derived from a well containing recombinant mCherry-sfGFP fusion protein and average fluorescence across the stack was calculated for each cell. Cells were classified as autofluorescence control or sample separately for each field of view by fitting a 2-component Gaussian mixture model to the mCherry intensity values and assigning each cell to the class with the higher posterior probability. GFP intensity of all control cells within a field of view was averaged and subtracted from the sample GFP intensities.

\section{Acknowledgments}

We acknowledge the support of Bernd Heßling (ZMBH Mass Spectrometry facility) and Monika Langlotz (ZMBH Flow Cytometry and FACS facility). We thank Birgit Besenbeck for technical support, Daniel Kirrmaier for support with fluorescence microscopy, Frauke Melchior and Marius Lemberg for critically reading the manuscript. Work was supported by the Deutsche Forschungsgemeinschaft (SFB1036) to MK and an MSc/PhD fellowship from the HBIGS graduate school to IK. 


\section{Author contributions}

MK conceived the project. MK, IK, MKs, AK, and TR designed the experiments and discussed the results. IK, MKs, LA, and TR performed the experiments. MK and IK wrote the manuscript.

\section{Conflict of interest}

The authors declare no conflict of interest.

\section{Supplementary information}

\section{Table S1: Yeast strains}

\begin{tabular}{|c|c|c|c|}
\hline $\begin{array}{l}\text { Strain } \\
\text { FY1679 }\end{array}$ & $\begin{array}{l}\text { Background } \\
\text { S288c }\end{array}$ & $\begin{array}{l}\text { Genotype } \\
\text { MATa/a ura3-52/ura3-52 leu2 } \Delta 1 / \text { LEU2 his } 3 \Delta 200 / \text { HIS3 trp1 } \Delta 63 / \text { TRP1 } \\
\text { GAL2/GAL2 }\end{array}$ & $\begin{array}{l}\text { Reference } \\
{[71]}\end{array}$ \\
\hline ESM356-1 & FY1679 & MATa ura3-52 leu2 $\Delta 1$ his $3 \Delta 200 \operatorname{trp} 1 \Delta 63$ & Elmar Schiebel \\
\hline YCT1084 & ESM356-1 & ubr1 $1 \Delta:$ hphNT1 & [72] \\
\hline YMaM632 & ESM356-1 & naa20 $\Delta:$ :hphNT1 & {$[12]$} \\
\hline YBB4 & ESM356-1 & ufd $4 \Delta:: h p h N T 1$ & [12] \\
\hline YBB5 & ESM356-1 & naa10 $\Delta:$ :hphNT1 & [12] \\
\hline YBB9 & ESM356-1 & ufd $4 \Delta::$ natNT2 ubr1 $\Delta::$ hphNT1 & {$[12]$} \\
\hline YBB52 & ESM356-1 & UFD4-3HA-kanMX6 & This study \\
\hline YBB53 & ESM356-1 & naa10 $\Delta:$ hphNT1 UFD4-3HA-kanMX6 & This study \\
\hline YEO2 & ESM356-1 & naa $10 \Delta::$ kanMX6 ubr1 $\Delta::$ hphNT1 & This study \\
\hline YEO3 & ESM356-1 & naa10 $\Delta:: k a n M X 6$ ufd $4 \Delta::$ natNT2 ubr1 $\Delta::$ hphNT1 & {$[12]$} \\
\hline YIK35 & ESM356-1 & naa10 $\Delta::$ kanMX6 ufd $4 \Delta::$ hphNT1 & This study \\
\hline YIK55 & ESM356-1 & ufd $2 \triangle:$ :kIURA3 & This study \\
\hline YIK56 & ESM356-1 & ufd $2 \Delta::$ kIURA3 ufd4 $\Delta::$ natNT2 ubr1 $\Delta::$ hphNT1 & This study \\
\hline YIK241 & ESM356-1 & ubr2 $\Delta::$ kIUra3 ufd $4 \Delta::$ natNT2 ubr1 $\Delta::$ hphNT1 & This study \\
\hline YIK242 & ESM356-1 & ubr2 $\Delta::$ kIUra3 ufd $4 \Delta::$ natNT2 ubr1 $\Delta::$ hphNT1 naa10 $\Delta::$ kanMX6 & This study \\
\hline YIK278 & ESM356-1 & tom $1 \Delta:: \mathrm{klU}$ ra3 & This study \\
\hline YIK280 & ESM356-1 & tom $1 \Delta::$ kIURA3 ufd $4 \Delta::$ natNT2 ubr1 $\Delta::$ hphNT1 & This study \\
\hline YIK281 & ESM356-1 & tom1 $\Delta:: k I U R A 3$ naa10 $\Delta:: k a n M X 6$ ufd $4 \Delta::$ natNT2 ubr1 $\Delta::$ hphNT1 & This study \\
\hline YIK292 & ESM356-1 & ufd $2 \Delta::$ natNT2 tom $1 \Delta::$ kIURA3 & This study \\
\hline YIK300 & ESM356-1 & tom $1 \Delta::$ natNT2 ubr1 $\Delta::$ hphNT1 & This study \\
\hline YIK301 & ESM356-1 & tom $1 \Delta::$ natNT2 ufd $4 \Delta::$ hphNT1 & This study \\
\hline YIK305 & ESM356-1 & TOM1-TAP-kanMX4 & This study \\
\hline YIK309 & ESM356-1 & RPN4-TAP-kanMX4 & This study \\
\hline YIK311 & ESM356-1 & naa10 $\Delta:$ natNT2 RPN4-TAP-kanMX4 & This study \\
\hline YIK330 & ESM356-1 & pep $4 \Delta 0$ & This study \\
\hline YIK343 & ESM356-1 & natNT2-pGPD-FLAG-UFD4 pep4 $\Delta 0$ & This study \\
\hline YIK344 & ESM356-1 & natNT2-pGPD-FLAG-TOM 1 pep $4 \Delta 0$ & This study \\
\hline YIK345 & ESM356-1 & natNT2-pGPD-FLAG-UBR1 pep4 $\Delta 0$ & This study \\
\hline YIK346 & ESM356-1 & natNT2-pGPD-FLAG-UFD2 pep $4 \Delta 0$ & This study \\
\hline YIK358 & ESM356-1 & pPRE4-sfGFP-KanMX-pPRE4-PRE4 & This study \\
\hline YIK359 & ESM356-1 & pPRE5-sfGFP-KanMX-pPRE5-PRE5 & This study \\
\hline YIK360 & ESM356-1 & pPRE6-sfGFP-KanMX-pPRE6-PRE6 & This study \\
\hline YIK361 & ESM356-1 & pRPT3-sfGFP-KanMX-pRPT3-RPT3 & This study \\
\hline YIK362 & ESM356-1 & pRPT5-sfGFP-KanMX-pRPT5-RPT5 & This study \\
\hline YIK363 & ESM356-1 & pPUP1-sfGFP-KanMX-pPUP1-PUP1 & This study \\
\hline YIK364 & ESM356-1 & pTUB1-sfGFP-KanMX-pTUB1-TUB1 & This study \\
\hline YIK366 & ESM356-1 & pRPB2-sfGFP-KanMX-pRPB2-RPB2 & This study \\
\hline YIK367 & ESM356-1 & naa $10 \Delta::$ natNT2 pPRE4-sfGFP-KanMX-pPRE4-PRE4 & This study \\
\hline YIK368 & ESM356-1 & naa $10 \Delta::$ natNT2 pPRE5-sfGFP-KanMX-pPRE5-PRE5 & This study \\
\hline YIK369 & ESM356-1 & naa $10 \triangle:$ :natNT2 pPRE6-sfGFP-KanMX-pPRE6-PRE6 & This study \\
\hline YIK370 & ESM356-1 & naa $10 \Delta::$ natNT2 pRPT3-sfGFP-KanMX-pRPT3-RPT3 & This study \\
\hline YIK371 & ESM356-1 & naa10 $\Delta::$ natNT2 pRPT5-sfGFP-KanMX-pRPT5-RPT5 & This study \\
\hline YIK372 & ESM356-1 & naa $10 \Delta:$ natNT2 pPUP1-sfGFP-KanMX-pPUP1-PUP1 & This study \\
\hline YIK373 & ESM356-1 & naa10 $\Delta::$ natNT2 pTUB1-sfGFP-KanMX-pTUB1-TUB1 & This study \\
\hline YIK375 & ESM356-1 & naa $10 \Delta::$ natNT2 pRPB2-sfGFP-KanMX-pRPB2-RPB2 & This study \\
\hline YIK385 & ESM356-1 & pPRE4-sfGFP-KanMX-pPRE4-PRE4 Rpn4A2N & This study \\
\hline YIK386 & ESM356-1 & pPRE5-sfGFP-KanMX-pPRE5-PRE5 Rpn4A2N & This study \\
\hline YIK387 & ESM356-1 & pPRE6-sfGFP-KanMX-pPRE6-PRE6 Rpn4A2N & This study \\
\hline YIK388 & ESM356-1 & pRPT3-sfGFP-KanMX-pRPT3-RPT3 Rpn4A2N & This study \\
\hline YIK389 & ESM356-1 & pRPT5-sfGFP-KanMX-pRPT5-RPT5 Rpn4A2N & This study \\
\hline YIK390 & ESM356-1 & pPUP1-sfGFP-KanMX-pPUP1-PUP1 Rpn4A2N & This study \\
\hline YIK391 & ESM356-1 & pTUB1-sfGFP-KanMX-pTUB1-TUB1 Rpn4A2N & This study \\
\hline
\end{tabular}




\begin{tabular}{|c|c|c|c|}
\hline Strain & Background & Genotype & Reference \\
\hline YIK393 & ESM356-1 & pRPB2-sfGFP-KanMX-pRPB2-RPB2 Rpn4A2N & This study \\
\hline YIK398 & ESM356-1 & naa10 $\Delta:$ :natNT2 pPRE4-sfGFP-KanMX-pPRE4-PRE4 Rpn4A2N & This study \\
\hline YIK399 & ESM356-1 & naa10 $\Delta:$ :natNT2 pPRE5-sfGFP-KanMX-pPRE5-PRE5 Rpn4A2N & This study \\
\hline YIK401 & ESM356-1 & naa10 $\Delta:$ natNT2 pRPT3-sfGFP-KanMX-pRPT3-RPT3 Rpn4A2N & This study \\
\hline YIK402 & ESM356-1 & naa10 $\Delta:$ :natNT2 pRPT5-sfGFP-KanMX-pRPT5-RPT5 Rpn4A2N & This study \\
\hline YIK403 & ESM356-1 & naa10 $\Delta:$ :natNT2 pPUP1-sfGFP-KanMX-pPUP1-PUP1 Rpn4A2N & This study \\
\hline YIK414 & ESM356-1 & ubr1 $\Delta:: \mathrm{klTrp} 1$ ufd4 $\Delta:: \mathrm{hphNT} 1 \mathrm{Rpn} 4 \mathrm{~A} 2 \mathrm{~N}$ & This study \\
\hline YIK415 & ESM356-1 & ubr1 $\Delta:: \mathrm{klTrp} 1$ ufd $4 \Delta::$ hphNT1 naa10 $\Delta::$ natNT2 Rpn4A2N & This study \\
\hline YIK423 & ESM356-1 & tom1 $\Delta:: k a n M X 6$ ufd4 $\Delta:: h p h N T 1$ ubr1 $\Delta:: k \mid T r p 1$ Rpn4A2N & This study \\
\hline YIK424 & ESM356-1 & tom $1 \Delta::$ kanMX6 naa10 $\Delta::$ natNT2 ufd4 $\Delta:$ hphNT1 ubr1 $\Delta:$ klTrp1 Rpn4A2N & This study \\
\hline YIK427 & ESM356-1 & Rpn4 $\Delta(211-229)-T A P-k a n M X 4$ & This study \\
\hline YIK432 & ESM356-1 & naa20 $\Delta:$ :natNT2 Rpn4A2N $\Delta(211-229)-T A P-k a n M X 4$ & This study \\
\hline YIK460 & ESM356-1 & ubr1 $\Delta::$ kanMX6 ufd4 $\Delta::$ natNT2 Rpn4 $\Delta(211-229)$ & This study \\
\hline YIK462 & ESM356-1 & ubr1 $\Delta:: \operatorname{kanMX6}$ ufd $4 \Delta::$ natNT2 naa10 $\Delta::$ hphNT1 Rpn4 $\Delta(211-229)$ & This study \\
\hline YIK464 & ESM356-1 & ubr2 $\Delta::$ kIUra3 ubr1 $\Delta:: k a n M X 6$ ufd4 $\Delta::$ natNT2 Rpn4 $\Delta(211-229)$ & This study \\
\hline YIK466 & ESM356-1 & $\begin{array}{l}\text { ubr2 } \Delta:: \text { klUra3 ubr1 } \Delta:: \text { kanMX6 ufd } 4 \Delta:: \text { natNT2 naa10 } \Delta: \text { hphNT1 Rpn4 } \Delta(211- \\
\text { 229) }\end{array}$ & This study \\
\hline YIK469 & ESM356-1 & $\operatorname{pdr} 5 \Delta:: \operatorname{kanMX6}$ ubr2 $\Delta::$ natNT2 & This study \\
\hline YIK470 & ESM356-1 & pdr5 $\Delta::$ kanMX6 ubr2 $\Delta::$ natNT2 naa10 $\Delta::$ hphNT1 & This study \\
\hline YIK471 & ESM356-1 & pdr5 $\Delta::$ kanMX6 ubr2 $\Delta::$ natNT2 naa20 $\Delta::$ hphNT1 & This study \\
\hline YIK476 & ESM356-1 & plK117 in YIK469 & This study \\
\hline YIK477 & ESM356-1 & plK118 in YIK469 & This study \\
\hline YIK478 & ESM356-1 & plK117 in YIK470 & This study \\
\hline YIK644 & ESM356-1 & leu2 $\Delta::$ pGPD-mCherry-tCYC1-hphNT1 TOM1-sfGFP-kanMX & This study \\
\hline YIK645 & ESM356-1 & leu2 $\Delta::$ pGPD-mCherry-tCYC1-hphNT1 naa10 $\triangle::$ natNT2 TOM1-sfGFP-kanMX & This study \\
\hline
\end{tabular}

\section{Table S2: Plasmids}

\begin{tabular}{|c|c|c|}
\hline Plasmid & Description & Reference \\
\hline pET28c & E. coli expression vector & Novagen \\
\hline pFA6a-kanMX6 & $\begin{array}{l}\text { template for gene deletion by PCR targeting with kanMX6 selection } \\
\text { marker }\end{array}$ & (Wach et al., 1994) \\
\hline pFA6a-hphNT1 & $\begin{array}{l}\text { template for gene deletion by PCR targeting with hphNT1 selection } \\
\text { marker }\end{array}$ & (Janke et al., 2004) \\
\hline pFA6a-natNT2 & template for gene deletion by PCR targeting with natNT2 selection marker & (Janke et al., 2004) \\
\hline pYM13 & $\begin{array}{l}\text { Template for C-terminal tagging with TAP-tag by PCR targeting with } \\
\text { kanMX6 selection marker }\end{array}$ & (Janke et al., 2004) \\
\hline pYM23 & $\begin{array}{l}\text { Template for C-terminal tagging with } 3 \text { Myc-tag by PCR targeting with } \\
\text { kITrp1 selection marker }\end{array}$ & (Janke et al., 2004) \\
\hline pRS413 & CEN ARS HIS3 & (Sikorski and Hieter) \\
\hline p413-GPD & CEN ARS HIS3 pGPD-tCYC1 & (Mumberg et al.) \\
\hline pArd1 & pRS416-NAA10 & Ulrike Friedrich \\
\hline pGR295 & p415-TEF-10xHis-Ubi & $\begin{array}{l}\text { (Khmelinskii et al., } \\
\text { 2014) }\end{array}$ \\
\hline pAnB19 & pRS413-pGPD-Ubi-EcoRV-STOP-e ${ }^{\mathrm{K}}$-mCherry-sfGFP & (Kats et al., 2018) \\
\hline pAnB19-PP & pRS413-pGPD-Ubi-PP-e ${ }^{\mathrm{k}}$-mCherry-sfGFP & (Kats et al., 2018) \\
\hline pAnB19-EH & pRS413-pGPD-Ubi-EH-e $\mathrm{K}^{\mathrm{K}}$-mCherry-sfGFP & (Kats et al., 2018) \\
\hline pAnB19-UbiG76V & pRS413-pGPD-Ubi ${ }^{G 76 V}-\mathrm{e}^{\mathrm{K}}$-mCherry-sfGFP & (Kats et al., 2018) \\
\hline plK35 & pFA6a-kIUra3 & (Kats et al., 2018) \\
\hline plK41 & pRS413-pGPD-Ubi ${ }^{G 76 V}-\mathrm{e}^{\mathrm{K}}-\mathrm{mCherry-sfGFPcp8}$ & This study \\
\hline plK45 & pRS413-pGPD-Ubi ${ }^{\mathrm{K} 48 R, G 76 \mathrm{~V}}-\mathrm{e}^{\mathrm{K}}$-mCherry-sfGFP & This study \\
\hline plK57 & pRS413-pGPD-Rpn4(1-80)-e $\mathrm{e}^{\mathrm{K}}$-mCherry-sfGFP & This study \\
\hline plK59 & $\begin{array}{l}\text { template for pGPD-driven overexpression and N-terminal tagging with } \\
\text { FLAG-tag by PCR targeting with natNT2 selection marker }\end{array}$ & This study \\
\hline plK66 & pRS413-pGPD-Rpn4 ${ }^{\mathrm{A} 2 \mathrm{~N}}(1-80)-\mathrm{e}^{\mathrm{K}}$-mCherry-sfGFP & This study \\
\hline plK78 & 6xHis-Ubc4 in pET28c & This study \\
\hline plK79 & 6xHis-Rad6 in pET28c & This study \\
\hline plK100 & Ubi-ProtA-6xHis in pET28c & This study \\
\hline plK102 & UbiK48R-ProtA-6xHis in pET28c & This study \\
\hline plK117 & p413-GPD-Rpn4 ${ }^{\text {C477A }}$-10xHis-sfGFPcp8 & This study \\
\hline plK118 & p413-GPD-Rpn4A2N ${ }^{\text {C477A-10xHis-sfGFPcp8 }}$ & This study \\
\hline
\end{tabular}




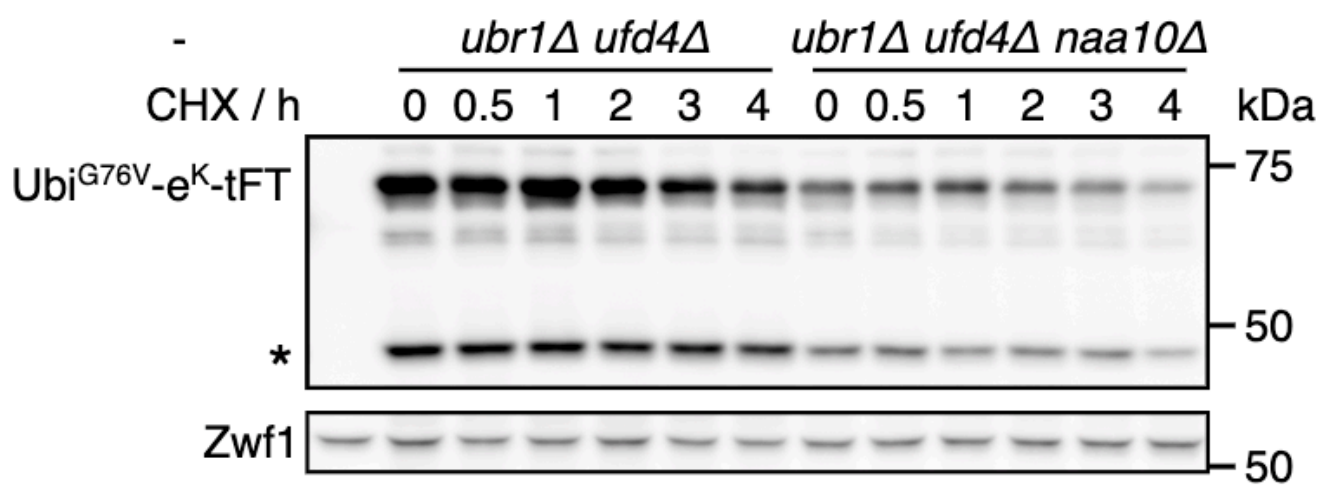

Fig. S1. Degradation of UbiG76V-tFT.

Degradation of UbiG76V-tFT after blocking translation with cycloheximide. Whole-cell extracts were separated by SDS-PAGE followed by immunoblotting with antibodies against GFP and Zwf1 as loading control. A product of mCherry hydrolysis during cell extract preparation (Gross et al., 2000) is marked (*).

A

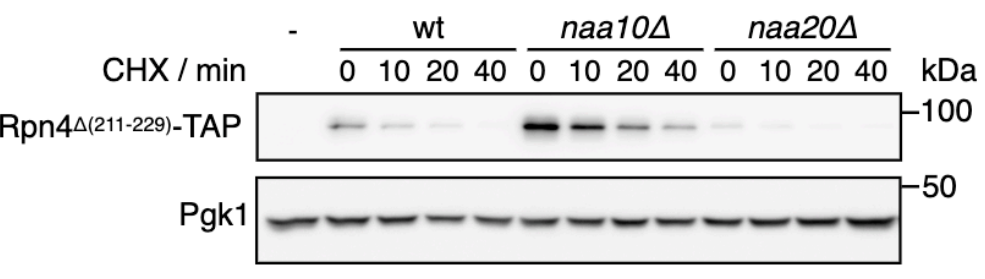

B
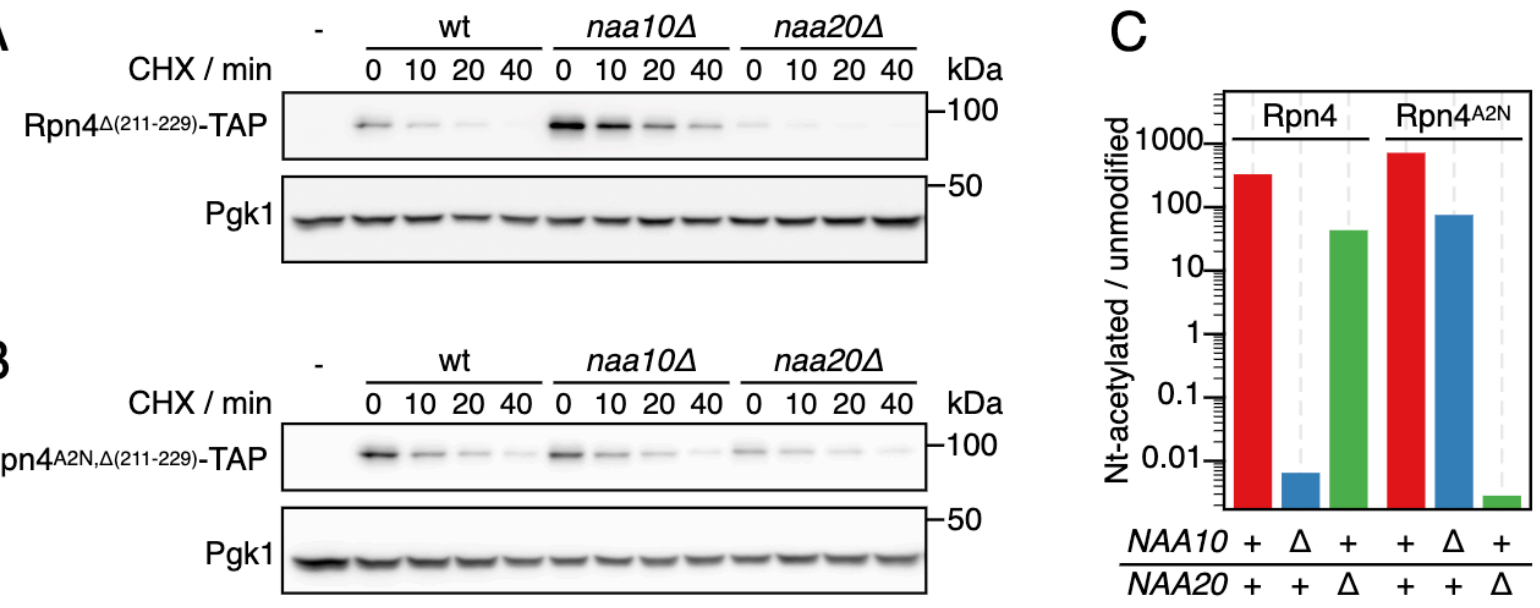

\section{S2 Fig. Degradation of Rpn4 variants.}

Cyclohexiide chase analysis of the degradation of Rpn4 variants. Whole-cell extracts were separated by SDS-PAGE followed by immunoblotting with antibodies against protein $A$ and Pgk1 as loading control.

(A) Rpn4 $\Delta$ (211-229) lacking the ubiquitin-dependent degron. Representative immunoblot from Fig $2 \mathrm{~F}$.

(B) Rpn4A2N, $\Delta(211-229)$ lacking the ubiquitin-dependent degron and acetylated by NatB instead of NatA. Representative immunoblot from Fig $2 \mathrm{~F}$. 
A

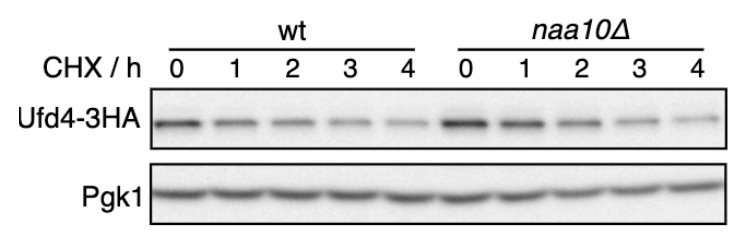

B

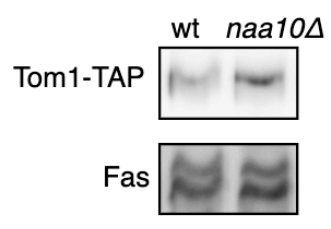

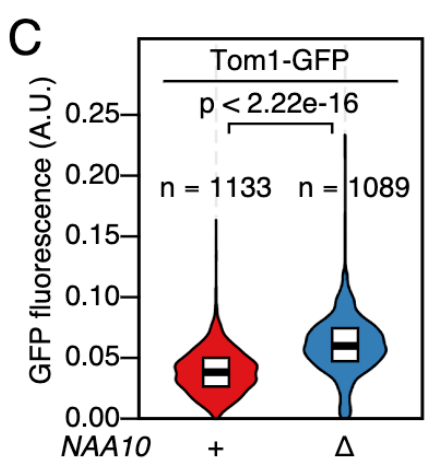

S3 Fig. E3 ligases in cells lacking NatA.

(A) Degradation of Ufd4 after blocking translation with cycloheximide. Whole-cell extracts were separated by SDS-PAGE followed by immunoblotting with antibodies against HA and Pgk1 as loading control. Representative immunoblot from Fig 5A. Time point 0 was used for quantification.

(B) Abundance of Tom1. Whole-cell extracts were separated by SDS-PAGE followed by immunoblotting with antibodies against protein $A$ and Fas as loading control. Representative immunoblot from Fig 5 A.

(C) Abundance of Tom1. Live-cell imaging of strains carrying C-terminally GFP-tagged Tom1 was performed and the fluorescence intensity was quantified. $p$ : Mann-Whitney U-test.

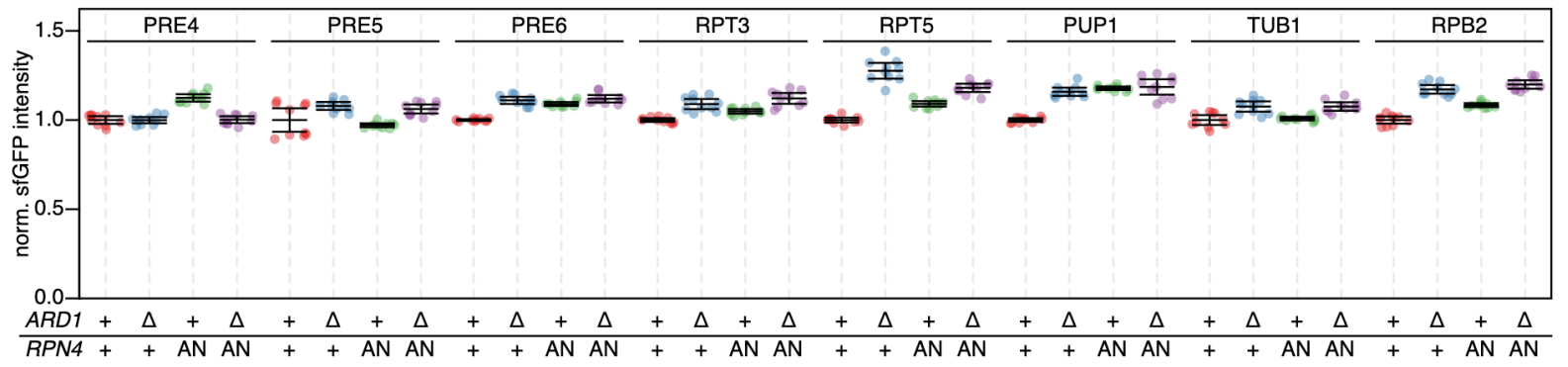

S4 Fig: Influence of NatA and Rpn4 on activity of proteasomal promoters.

Promoters of the indicated genes were duplicated while simultaneously inserting a sfGFP coding sequence, such that expression of sfGFP is driven by the second copy of the promoter. Fluorescence intensity was measured by flow cytometry and normalized to wild type cells. Mean intensity and $95 \% \mathrm{Cl}$ of six replicates are plotted together with the intensity of each replicate. The TUB1 and RPB2 promoters served as Rpn4-independent controls.

\section{References}

Aksnes, H., A. Drazic, M. Marie, and T. Arnesen. 2016. First Things First: Vital Protein Marks by N-Terminal Acetyltransferases. Trends Biochem. Sci. 41:746-760. 
Alexey, I.N., K. Andrew, a. Eugene Kolker, and R. Aebersold. 2003. A Statistical Model for Identifying Proteins by Tandem Mass Spectrometry. Anal. Chem. 75:4646-4658.

Amerik, A.Y., and M. Hochstrasser. 2004. Mechanism and function of deubiquitinating enzymes. Biochimica et Biophysica Acta (BBA) - Molecular Cell Research. 1695:189207.

Babiano, R., M. Gamalinda, J.L. Woolford, and J. de la Cruz. 2012. Saccharomyces cerevisiae ribosomal protein L26 is not essential for ribosome assembly and function. Mol. Cell. Biol. 32:3228-3241.

Bachmair, A., D. Finley, and A. Varshavsky. 1986. In vivo half-life of a protein is a function of its amino-terminal residue. Science. 234:179-186.

Bachmair, A., and A. Varshavsky. 1989. The degradation signal in a short-lived protein. Cell. 56:1019-1032.

Bloom, J., V. Amador, F. Bartolini, G. DeMartino, and M. Pagano. 2003. Proteasome-mediated degradation of p21 via N-terminal ubiquitinylation. Cell. 115:71-82.

Caron, C., C. Boyault, and S. Khochbin. 2005. Regulatory cross-talk between lysine acetylation and ubiquitination: role in the control of protein stability. Bioessays. 27:408-415.

Coulombe, P., G. Rodier, E. Bonneil, P. Thibault, and S. Meloche. 2004. N-Terminal ubiquitination of extracellular signal-regulated kinase 3 and p21 directs their degradation by the proteasome. Mol. Cell. Biol. 24:6140-6150.

Cox, J., and M. Mann. 2008. MaxQuant enables high peptide identification rates, individualized p.p.b.-range mass accuracies and proteome-wide protein quantification. Nat. Biotechnol. 26:1367-1372.

Dantuma, N.P., K. Lindsten, R. Glas, M. Jellne, and M.G. Masucci. 2000. Short-lived green fluorescent proteins for quantifying ubiquitin/proteasome-dependent proteolysis in living cells. Nat. Biotechnol. 18:538-543.

Defenouillère, Q., Y. Yao, J. Mouaikel, A. Namane, A. Galopier, L. Decourty, A. Doyen, C. Malabat, C. Saveanu, A. Jacquier, and M. Fromont-Racine. 2013. Cdc48-associated complex bound to $60 \mathrm{~S}$ particles is required for the clearance of aberrant translation products. Proc. Natl. Acad. Sci. U.S.A. 110:5046-5051.

Egner, R., M. Thumm, M. Straub, A. Simeon, H.J. Schüller, and D.H. Wolf. 1993. Tracing intracellular proteolytic pathways. Proteolysis of fatty acid synthase and other cytoplasmic proteins in the yeast Saccharomyces cerevisiae. J. Biol. Chem. 268:27269-27276.

Fecher-Trost, C., U. Wissenbach, A. Beck, P. Schalkowsky, C. Stoerger, J. Doerr, A. Dembek, M. Simon-Thomas, A. Weber, P. Wollenberg, T. Ruppert, R. Middendorff, H.H. Maurer, and V. Flockerzi. 2013. The in vivo TRPV6 protein starts at a non-AUG triplet, decoded as methionine, upstream of canonical initiation at AUG. Journal of Biological Chemistry. 288:16629-16644.

Fiil, B.K., R.B. Damgaard, S.A. Wagner, K. Keusekotten, M. Fritsch, S. Bekker-Jensen, N. Mailand, C. Choudhary, D. Komander, and M. Gyrd-Hansen. 2013. OTULIN Restricts Met1-Linked Ubiquitination to Control Innate Immune Signaling. Mol. Cell. 50:818-830.

Finley, D., H.D. Ulrich, T. Sommer, and P. Kaiser. 2012. The ubiquitin-proteasome system of Saccharomyces cerevisiae. Genetics. 192:319-360.

Friedrich, U.A., M. Zedan, B. Hessling, K. Fenzl, L. Gillet, J.D. Barry, M. Knop, G. Kramer, and B. Bukau. Na-terminal acetylation of proteins by NatA and NatB serves distinct physiological roles in Saccharomyces cerevisiae. biorxiv.org.

Gawron, D., E. Ndah, K. Gevaert, and P. Van Damme. 2016. Positional proteomics reveals differences in N-terminal proteoform stability. Mol. Syst. Biol. 12:858. 
Gerlach, B., S.M. Cordier, A.C. Schmukle, C.H. Emmerich, E. Rieser, T.L. Haas, A.I. Webb, J.A. Rickard, H. Anderton, W.W.L. Wong, U. Nachbur, L. Gangoda, U. Warnken, A.W. Purcell, J. Silke, and H. Walczak. 2011. Linear ubiquitination prevents inflammation and regulates immune signalling. Nature. 471:591-596.

Gross, L.A., G.S. Baird, R.C. Hoffman, K.K. Baldridge, and R.Y. Tsien. 2000. The structure of the chromophore within DsRed, a red fluorescent protein from coral. Proc. Natl. Acad. Sci. U.S.A. 97:11990-11995.

Ha, S.-W., D. Ju, and Y. Xie. 2012. The N-terminal domain of Rpn4 serves as a portable ubiquitin-independent degron and is recognized by specific 19S RP subunits. Biochem. Biophys. Res. Commun. 419:226-231.

Hahn, J.S., D.W. Neef, and D.J. Thiele. 2006. A stress regulatory network for co-ordinated activation of proteasome expression mediated by yeast heat shock transcription factor. Mol. Microbiol. 60:240-251.

Hershko, A., and A. Ciechanover. 1998. The ubiquitin system. Annu. Rev. Biochem. 67:425479.

Hershko, A., H. Heller, E. Eytan, G. Kaklij, and I.A. Rose. 1984. Role of the alpha-amino group of protein in ubiquitin-mediated protein breakdown. Proc. Natl. Acad. Sci. U.S.A. 81:7021-7025.

Horecka, J., and R.W. Davis. 2014. The 50:50 method for PCR-based seamless genome editing in yeast. Yeast. 31:103-112.

Huber, F., M. Meurer, D. Bunina, I. Kats, C.I. Maeder, M. Stefl, C. Mongis, and M. Knop. 2014. PCR Duplication: A One-Step Cloning-Free Method to Generate Duplicated Chromosomal Loci and Interference-Free Expression Reporters in Yeast. 9:e114590.

Hwang, C.-S., A. Shemorry, D. Auerbach, and A. Varshavsky. 2010a. The N-end rule pathway is mediated by a complex of the RING-type Ubr1 and HECT-type Ufd4 ubiquitin ligases. Nat. Cell Biol. 12:1177-1185.

Hwang, C.-S., A. Shemorry, and A. Varshavsky. 2009. Two proteolytic pathways regulate DNA repair by cotargeting the Mgt1 alkylguanine transferase. Proceedings of the National Academy of Sciences. 106:2142-2147.

Hwang, C.-S., A. Shemorry, and A. Varshavsky. 2010b. N-terminal acetylation of cellular proteins creates specific degradation signals. Science. 327:973-977.

Hwang, C.-S., and A. Varshavsky. 2008. Regulation of peptide import through phosphorylation of Ubr1, the ubiquitin ligase of the N-end rule pathway. Proc. Natl. Acad. Sci. U.S.A. 105:19188-19193.

Janke, C., M.M. Magiera, N. Rathfelder, C. Taxis, S. Reber, H. Maekawa, A. Moreno-Borchart, G. Doenges, E. Schwob, E. Schiebel, and M. Knop. 2004. A versatile toolbox for PCRbased tagging of yeast genes: new fluorescent proteins, more markers and promoter substitution cassettes. Yeast. 21:947-962.

Johnson, E.S., B. Bartel, W. Seufert, and A. Varshavsky. 1992. Ubiquitin as a degradation signal. EMBO J. 11:497-505.

Johnson, E.S., P.C. Ma, I.M. Ota, and A. Varshavsky. 1995. A proteolytic pathway that recognizes ubiquitin as a degradation signal. J. Biol. Chem. 270:17442-17456.

Ju, D., L. Wang, X. Mao, and Y. Xie. 2004. Homeostatic regulation of the proteasome via an Rpn4-dependent feedback circuit. Biochem. Biophys. Res. Commun. 321:51-57.

Ju, D., and Y. Xie. 2004. Proteasomal degradation of RPN4 via two distinct mechanisms, ubiquitin-dependent and-independent. Journal of Biological Chemistry. 279:2385123854. 
Ju, D., and Y. Xie. 2006. Identification of the preferential ubiquitination site and ubiquitindependent degradation signal of Rpn4. J. Biol. Chem. 281:10657-10662.

Kats, I., A. Khmelinskii, M. Kschonsak, F. Huber, R.A. Knieß, A. Bartosik, and M. Knop. 2018. Mapping Degradation Signals and Pathways in a Eukaryotic N-terminome. Mol. Cell. 70:488-501.e485.

Keller, A., A.I. Nesvizhskii, E. Kolker, and R. Aebersold. 2002. Empirical Statistical Model To Estimate the Accuracy of Peptide Identifications Made by MS/MS and Database Search. Anal. Chem. 74:5383-5392.

Khmelinskii, A., E. Blaszczak, M. Pantazopoulou, B. Fischer, D.J. Omnus, G. Le Dez, A. Brossard, A. Gunnarsson, J.D. Barry, M. Meurer, D. Kirrmaier, C. Boone, W. Huber, G. Rabut, P.O. Ljungdahl, and M. Knop. 2014. Protein quality control at the inner nuclear membrane. Nature. 516:410-413.

Khmelinskii, A., P.J. Keller, A. Bartosik, M. Meurer, J.D. Barry, B.R. Mardin, A. Kaufmann, S. Trautmann, M. Wachsmuth, G. Pereira, W. Huber, E. Schiebel, and M. Knop. 2012. Tandem fluorescent protein timers for in vivo analysis of protein dynamics. Nat. Biotechnol. 30:708-714.

Khmelinskii, A., and M. Knop. 2014. Analysis of protein dynamics with tandem fluorescent protein timers. Methods Mol. Biol. 1174:195-210.

Khmelinskii, A., M. Meurer, C.-T. Ho, B. Besenbeck, J. Füller, M.K. Lemberg, B. Bukau, A. Mogk, and M. Knop. 2016. Incomplete proteasomal degradation of green fluorescent proteins in the context of tandem fluorescent protein timers. Mol. Biol. Cell. 27:360-370.

Kim, D.-H., and D.M. Koepp. 2012. Hect E3 ubiquitin ligase Tom1 controls Dia2 degradation during the cell cycle. Mol. Biol. Cell. 23:4203-4211.

Kim, D.-H., W. Zhang, and D.M. Koepp. 2012. The Hect domain E3 ligase Tom1 and the Fbox protein Dia2 control Cdc6 degradation in G1 phase. Journal of Biological Chemistry. 287:44212-44220.

Knop, M., K. Siegers, G. Pereira, W. Zachariae, B. Winsor, K. Nasmyth, and E. Schiebel. 1999. Epitope tagging of yeast genes using a PCR-based strategy: more tags and improved practical routines. Yeast. 15:963-972.

Koegl, M., T. Hoppe, S. Schlenker, H.D. Ulrich, T.U. Mayer, and S. Jentsch. 1999. A novel ubiquitination factor, E4, is involved in multiubiquitin chain assembly. Cell. 96:635-644.

Kuo, M.-L., W. den Besten, D. Bertwistle, M.F. Roussel, and C.J. Sherr. 2004. N-terminal polyubiquitination and degradation of the Arf tumor suppressor. Genes Dev. 18:18621874.

Linster, E., I. Stephan, W.V. Bienvenut, J. Maple-Grødem, L.M. Myklebust, M. Huber, M. Reichelt, C. Sticht, S.G. Møller, T. Meinnel, T. Arnesen, C. Giglione, R. Hell, and M. Wirtz. 2015. Downregulation of $\mathrm{N}$-terminal acetylation triggers ABA-mediated drought responses in Arabidopsis. Nat Commun. 6:7640.

Liu, C., W. Liu, Y. Ye, and W. Li. 2017. Ufd2p synthesizes branched ubiquitin chains to promote the degradation of substrates modified with atypical chains. Nat Commun. 8:14274.

Mannhaupt, G., R. Schnall, V. Karpov, I. Vetter, and H. Feldmann. 1999. Rpn4p acts as a transcription factor by binding to PACE, a nonamer box found upstream of $26 \mathrm{~S}$ proteasomal and other genes in yeast. FEBS Lett. 450:27-34.

Mayer, C., S. Dimopoulos, F. Rudolf, and J. Stelling. 2013. Using CellX to quantify intracellular events. Curr Protoc Mol Biol. Chapter 14:Unit 14.22.

McDowell, G.S., and A. Philpott. 2013. Non-canonical ubiquitylation: mechanisms and consequences. Int. J. Biochem. Cell Biol. 45:1833-1842. 
Michel, M.A., K.N. Swatek, M.K. Hospenthal, and D. Komander. 2017. Ubiquitin LinkageSpecific Affimers Reveal Insights into K6-Linked Ubiquitin Signaling. Mol. Cell. 68:233246.e235.

Miller, S.B., C.T. Ho, J. Winkler, M. Khokhrina, A. Neuner, M.Y. Mohamed, D.L. Guilbride, K. Richter, M. Lisby, E. Schiebel, A. Mogk, and B. Bukau. 2015. Compartment-specific aggregases direct distinct nuclear and cytoplasmic aggregate deposition. EMBO J. 34:778-797.

Mumberg, D., R. Müller, and M. Funk. 1995. Yeast vectors for the controlled expression of heterologous proteins in different genetic backgrounds. Gene. 156:119-122.

Nguyen, K.T., S.-H. Mun, C.-S. Lee, and C.-S. Hwang. 2018. Control of protein degradation by $\mathrm{N}$-terminal acetylation and the $\mathrm{N}$-end rule pathway. Exp. Mol. Med. 50:91.

Noy, T., O. Suad, D. Taglicht, and A. Ciechanover. 2012. HUWE1 ubiquitinates MyoD and targets it for proteasomal degradation. Biochem. Biophys. Res. Commun. 418:408413.

Oh, J.-H., J.-Y. Hyun, and A. Varshavsky. 2017. Control of Hsp90 chaperone and its clients by $\mathrm{N}$-terminal acetylation and the $\mathrm{N}$-end rule pathway. Proceedings of the National Academy of Sciences. 157:201705898.

P de Bie, A.C. 2011. Ubiquitination of E3 ligases: self-regulation of the ubiquitin system via proteolytic and non-proteolytic mechanisms. Cell Death Differ. 18:1393-1402.

Park, Y., S.K. Yoon, and J.-B. Yoon. 2009. The HECT domain of TRIP12 ubiquitinates substrates of the ubiquitin fusion degradation pathway. J. Biol. Chem. 284:1540-1549.

Poulsen, E.G., C. Steinhauer, M. Lees, A.-M. Lauridsen, L. Ellgaard, and R. HartmannPetersen. 2012. HUWE1 and TRIP12 collaborate in degradation of ubiquitin-fusion proteins and misframed ubiquitin. PLoS ONE. 7:e50548.

Rao, H., F. Uhlmann, K. Nasmyth, and A. Varshavsky. 2001. Degradation of a cohesin subunit by the $\mathrm{N}$-end rule pathway is essential for chromosome stability. Nature. 410:955-959.

Ravid, T., and M. Hochstrasser. 2008. Diversity of degradation signals in the ubiquitinproteasome system. Nat. Rev. Mol. Cell Biol. 9:679-690.

Rittinger, K., and F. Ikeda. 2017. Linear ubiquitin chains: enzymes, mechanisms and biology. Open Biology. 7:170026.

Rothbauer, U., K. Zolghadr, S. Muyldermans, A. Schepers, M.C. Cardoso, and H. Leonhardt. 2008. A Versatile Nanotrap for Biochemical and Functional Studies with Fluorescent Fusion Proteins. Molecular I\& Cellular Proteomics. 7:282-289.

Scaglione, K.M., V. Basrur, N.S. Ashraf, J.R. Konen, K.S.J. Elenitoba-Johnson, S.V. Todi, and H.L. Paulson. 2013. The Ubiquitin-conjugating Enzyme (E2) Ube2w Ubiquitinates the N Terminus of Substrates. J. Biol. Chem. 288:18784-18788.

Schneider, C.A., W.S. Rasband, and K.W. Eliceiri. 2012. NIH Image to ImageJ: 25 years of image analysis. Nat Methods. 9:671-675.

Shemorry, A., C.-S. Hwang, and A. Varshavsky. 2013. Control of protein quality and stoichiometries by $\mathrm{N}$-terminal acetylation and the $\mathrm{N}$-end rule pathway. Mol. Cell. 50:540-551.

Shirozu, R., H. Yashiroda, and S. Murata. 2015. Identification of minimum Rpn4-responsive elements in genes related to proteasome functions. FEBS Lett. 589:933-940.

Sikorski, R.S., and P. Hieter. 1989. A system of shuttle vectors and yeast host strains designed for efficient manipulation of DNA in Saccharomyces cerevisiae. Genetics. 122:19-27. 
Singh, R.K., M. Gonzalez, M.H. Kabbaj, and A. Gunjan. 2012. Novel E3 ubiquitin ligases that regulate histone protein levels in the budding yeast Saccharomyces cerevisiae. PLoS ONE. 7:e36295.

Stack, J.H., M. Whitney, S.M. Rodems, and B.A. Pollok. 2000. A ubiquitin-based tagging system for controlled modulation of protein stability. Nat. Biotechnol. 18:1298-1302.

Starheim, K.K., K. Gevaert, and T. Arnesen. 2012. Protein N-terminal acetyltransferases: when the start matters. Trends Biochem. Sci. 37:152-161.

Sung, M.-K., T.R. Porras-Yakushi, J.M. Reitsma, F.M. Huber, M.J. Sweredoski, A. Hoelz, S. Hess, and R.J. Deshaies. 2016. A conserved quality-control pathway that mediates degradation of unassembled ribosomal proteins. eLife. 5:3429.

Tatham, M.H., A. Plechanovová, E.G. Jaffray, H. Salmen, and R.T. Hay. 2013. Ube2W conjugates ubiquitin to a-amino groups of protein N-termini. Biochemical Journal. 453:137-145.

Tokunaga, F., S.-i. Sakata, Y. Saeki, Y. Satomi, T. Kirisako, K. Kamei, T. Nakagawa, M. Kato, S. Murata, S. Yamaoka, M. Yamamoto, S. Akira, T. Takao, K. Tanaka, and K. Iwai. 2009. Involvement of linear polyubiquitylation of NEMO in NF-KB activation. Nat. Cell Biol. 11:123-132.

Tsuchiya, H., K. Tanaka, and Y. Saeki. 2013. The parallel reaction monitoring method contributes to a highly sensitive polyubiquitin chain quantification. Biochem. Biophys. Res. Commun. 436:223-229.

van Leeuwen, F.W., D.P.V. de Kleijn, H.H. van den Hurk, A. Neubauer, M.A.F. Sonnemans, J.A. Sluijs, S. Köycü, R.D.J. Ramdjielal, A. Salehi, G.J.M. Martens, F.G. Grosveld, J.P.H. Burbach, and E.M. Hol. 1998. Frameshift Mutants of $\beta$ Amyloid Precursor Protein and Ubiquitin-B in Alzheimer's and Down Patients. Science. 279:242-247.

Varland, S., H. Aksnes, F. Kryuchkov, F. Impens, D. Van Haver, V. Jonckheere, M. Ziegler, K. Gevaert, P. Van Damme, and T. Arnesen. 2018. N-terminal Acetylation Levels Are Maintained During Acetyl-CoA Deficiency in Saccharomyces cerevisiae. Mol. Cell Proteomics. 17:2309-2323.

Varshavsky, A. 2019. N-degron and C-degron pathways of protein degradation. Proceedings of the National Academy of Sciences. 116:358-366.

Vittal, V., L. Shi, D.M. Wenzel, K.M. Scaglione, E.D. Duncan, V. Basrur, K.S.J. ElenitobaJohnson, D. Baker, H.L. Paulson, P.S. Brzovic, and R.E. Klevit. 2014. Intrinsic disorder drives N-terminal ubiquitination by Ube2w. Nat. Chem. Biol. 11:83-89.

Wach, A., A. Brachat, R. Pöhlmann, and P. Philippsen. 1994. New heterologous modules for classical or PCR-based gene disruptions in Saccharomyces cerevisiae. Yeast. 10:1793-1808.

Wang, L., X. Mao, D. Ju, and Y. Xie. 2004a. Rpn4 is a physiological substrate of the Ubr2 ubiquitin ligase. J. Biol. Chem. 279:55218-55223.

Wang, X., J.J. Connelly, C.-L. Wang, and R. Sternglanz. 2004b. Importance of the Sir3 N Terminus and Its Acetylation for Yeast Transcriptional Silencing. Genetics. 168:547551.

Wang, X., H. Xu, S.W. Ha, D. Ju, and Y. Xie. 2010. Proteasomal Degradation of Rpn4 in Saccharomyces cerevisiae Is Critical for Cell Viability Under Stressed Conditions. Genetics. 184:335-342.

Xie, Y., and A. Varshavsky. 2001. RPN4 is a ligand, substrate, and transcriptional regulator of the 26S proteasome: a negative feedback circuit. Proc. Natl. Acad. Sci. U.S.A. 98:3056-3061. 
Yau, R.G., K. Doerner, E.R. Castellanos, D.L. Haakonsen, A. Werner, N. Wang, X.W. Yang, N. Martinez-Martin, M.L. Matsumoto, V.M. Dixit, and M. Rape. 2017. Assembly and Function of Heterotypic Ubiquitin Chains in Cell-Cycle and Protein Quality Control. Cell. 171:918-933.e920.

Zattas, D., D.J. Adle, E.M. Rubenstein, and M. Hochstrasser. 2013. N-terminal acetylation of the yeast Derlin Der1 is essential for Hrd1 ubiquitin-ligase activity toward luminal ER substrates. Mol. Biol. Cell. 24:890-900. 